\title{
The influence of contrast and spatial factors in the perceived shape of boundaries
}

\author{
SERGIO RONCATO and CLARA CASCO \\ Università di Padova, Padova, Italy
}

\begin{abstract}
When an edge can be perceived to continue either with a collinear edge of the opposite contrast polarity or with a noncollinear edge of the same contrast polarity, observers perceive an alignment between the edges of the same contrast polarity, even though they are noncollinear. Using several stimulus configurations and both free and tachistoscopic viewing, we studied the luminance and spatial factors affecting the perceived distortion and binding. The results showed that the two noncollinear edges tended to align when they had the same contrast polarity (Experiment 1A) and to misalign when they had opposite contrast polarity (Experiment 2), providing that (1) they were separated by a distance larger than 1 arcmin and smaller than 3-4 arcmin (for all configurations) and (2) they laterally overlapped for about 7 arcmin (Experiment 1B). The results also showed that the direction of apparent distortion depended on the direction of overlapping. The results of Experiment 3 ruled out the local attraction/repulsion explanation but, instead, supported the suggestion that the interaction concerned the global edges, or part of them, and produced an inward tilt, which made the edges of the same contrast polarity perceptually to align, or an outward tilt, so that the edges of opposite contrast polarity were perceived to be more misaligned. From the overlap and distance limits found, it can be inferred that for two noncollinear contours to join perceptually, the tilt must not exceed $18^{\circ}$, a limit compatible with the orientation bandwidth of contrast-sensitive early cortical mechanisms.
\end{abstract}

Many psychophysical and physiological studies have shown that the visual system is capable of integrating spatially separate contour segments, an operation resulting in the perception of continuity of contours and boundaries. We can distinguish two main directions in the research on the mechanisms underlying this phenomenon. In some studies, the mechanisms producing the binding of contour fragments have been analyzed directly. For example, observers are required to indicate whether they see a path of aligned elements within a group of small separate elements (Field, Hayes, \& Hess, 1993). In other studies, binding has been studied indirectly as a manifestation of a general phenomenon of object interaction, which is revealed in spatial facilitation (Dresp, 1999) - that is, enhancement of the detectability of a nearby stimulus. Several factors have been shown to affect the perception of boundary continuity: the alignment of fragments (the "relatability" of Kellman \& Shipley, 1991; Gilchrist, Humphreys, Riddoch, \& Neumann, 1997) and their color, luminance, and contrast (McIlhagga $\&$ Mullen, 1996). One of these variables, the contrast polarity, has caught our interest and is investigated in this article. Some researchers have assessed the role of this factor on grouping. McIlhagga and Mullen observed that element integration is much less successful when the

Correspondence concerning this article should be addressed to S. Roncato, Dipartimento di Psicologia Generale, Università di Padova, Via Venezia 8, 35100 Padova, Italy (e-mail: sergio.roncato@unipd.it). fragments alternate between positive and negative luminance contrast. Gilchrist et al. have demonstrated that the polarity contrast difference between paired items does not disrupt grouping. Field, Hayes, and Hess (2000) found that observers present significantly lower sensitivity, when asked to integrate elements in a path, when these are in alternating phase (opposite contrast), although they still performed significantly above chance. Thus, a phase-sensitive process may play a role in contour integration. Studies on spatial facilitation have demonstrated that high-contrast-inducing lines facilitate the detection of a target line of either contrast polarity when the distance separating them is wider than 20 arcmin (Wehrhahn \& Dresp, 1998). Experiments with edge-like inducers and collinear line targets demonstrated detection facilitation with shorter spatial separations, but only in conditions in which the inducers and the target had the same contrast polarity (Dresp \& Grossberg, 1997). Clearly, the role of contrast polarity in contour integration is still an open question. Many studies have led to the suggestion put forward by Dresp (1999) that "the disposition of the visual system to integrate contour information across relatively small spatial gaps is therefore clearly selective to the contrast sign carried by the collinear contour segments" (Dresp, 1999, p. 135). The problem of contrast polarity in contour integration will be addressed in the present article. In particular, we investigated the effect of contrast polarity on the integration of noncollinear contours.

Our research has been stimulated by the simple observation that, in contour integration, contrast polarity may 

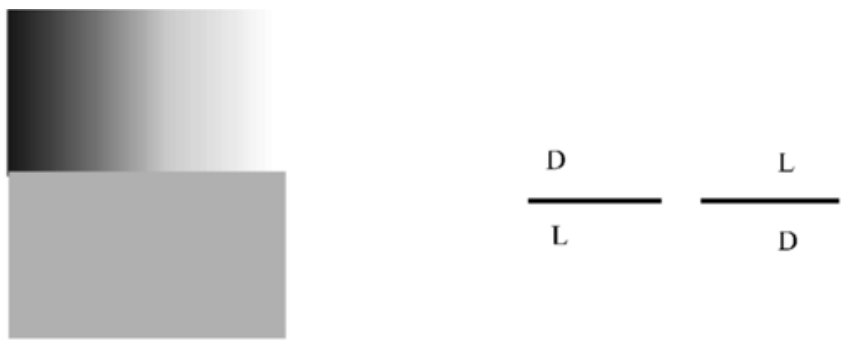

B
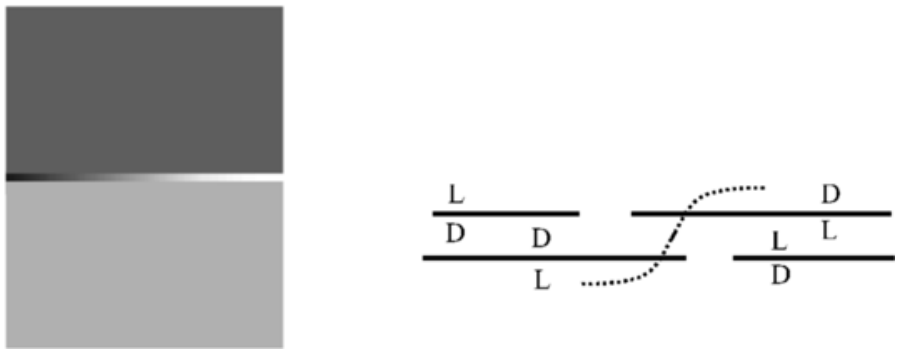

Figure 1. (A) A continuous straight edge appears to separate the two regions even if it is interrupted in the central region, where the upper and the lower areas are isoluminant. (B) As in panel A, but with the variable-shading area reduced to a thin strip, illustrated in the outline to the right, the perceived distortion that occurs when noncollinear edges of the same contrast polarity, laterally overlapping, join together. $D$ and $L$ indicate, respectively, the darker and the lighter of the two adjacent areas.

have the same binding strength as collinearity. Figure 1A depicts two contiguous areas; a uniform gray area below an area in shades from black to white. The edge configuration formed by this pattern can be illustrated, as in the outline on the right of Figure 1A, as two collinear edges of opposite contrast polarity: a dark-light (D/L) edge between the variable-shaded and uniform rectangles on the left half and a light-dark (L/D) edge on the right half, interrupted by a region in which the variable-shaded rectangle is isoluminant with the lower rectangle. Despite this interruption, the observer perceives a continuous straight edge between the two regions. This is considered a demonstration that the boundary completion process is insensitive to direction of contrast (Cohen \& Grossberg, 1984; Grossberg \& Mingolla, 1985). Nevertheless, identical edges in the same spatial arrangement can be perceived in a different way if presented in a more complex edge configuration. Figure 1B partly reproduces Figure 1A, with the variable-shaded region reduced in height, appearing as a thin strip. In this pattern, two pairs of collinear edges of opposite contrast polarity are present (illustrated in the outline on the right as $\mathrm{L} / \mathrm{D}-\mathrm{D} / \mathrm{L}$ above and $\mathrm{D} / \mathrm{L}-\mathrm{L} / \mathrm{D}$ below), and one would expect to perceive both pairs of collinear edges to bind. However, when asked to indicate whether a straight or a slanted contour is perceived, observers agree in describing it as deviating from straightness in the central zone. Our hypothesis is that in areas where two close edges run parallel, some integration may emerge that leads to the conjunction of edges of the same contrast polarity, althoughnoncollinear. This illusory slanting can be appreciated by observing the figures in panel $\mathrm{B}$ at a longer distance-for example, $1.5 \mathrm{~m}$. In this configuration, as in all the configurations used, contrast polarity is defined as illustrated in Figure 2.

What is the origin of the phenomenon? The perceptual system's attempts to join noncollinear edges would seem to be the obvious explanation for the origin of this perceptual slant. The location and direction of the apparent distortion may derive from the spatial arrangement between edges in the central region, as illustrated in the outline of Figure 3A. This outline illustrates that in this region, Collinear Edges 1 and 2 have opposite contrast polarity, Collinear Edges 3 and 4 have opposite contrast polarity, and NoncollinearEdges 1 and 4 and Noncollinear Edges 2 and 3 have the same contrast polarity, and Noncollinear Edges 1 and 4 run parallel in the central region (hereafter called the condition of lateral overlapping of noncollinear edges).

We speculate that this spatial arrangement may be crucial to perception of the clockwise slant indicated by the dotted line in the outline in Figure 3A. On the basis of this hypothesis, the prediction could be made that two noncollinear edges will tend to join when the following conditions occur: (1) They have the same contrast polarity, (2) their distance, calculated perpendicularly to the 


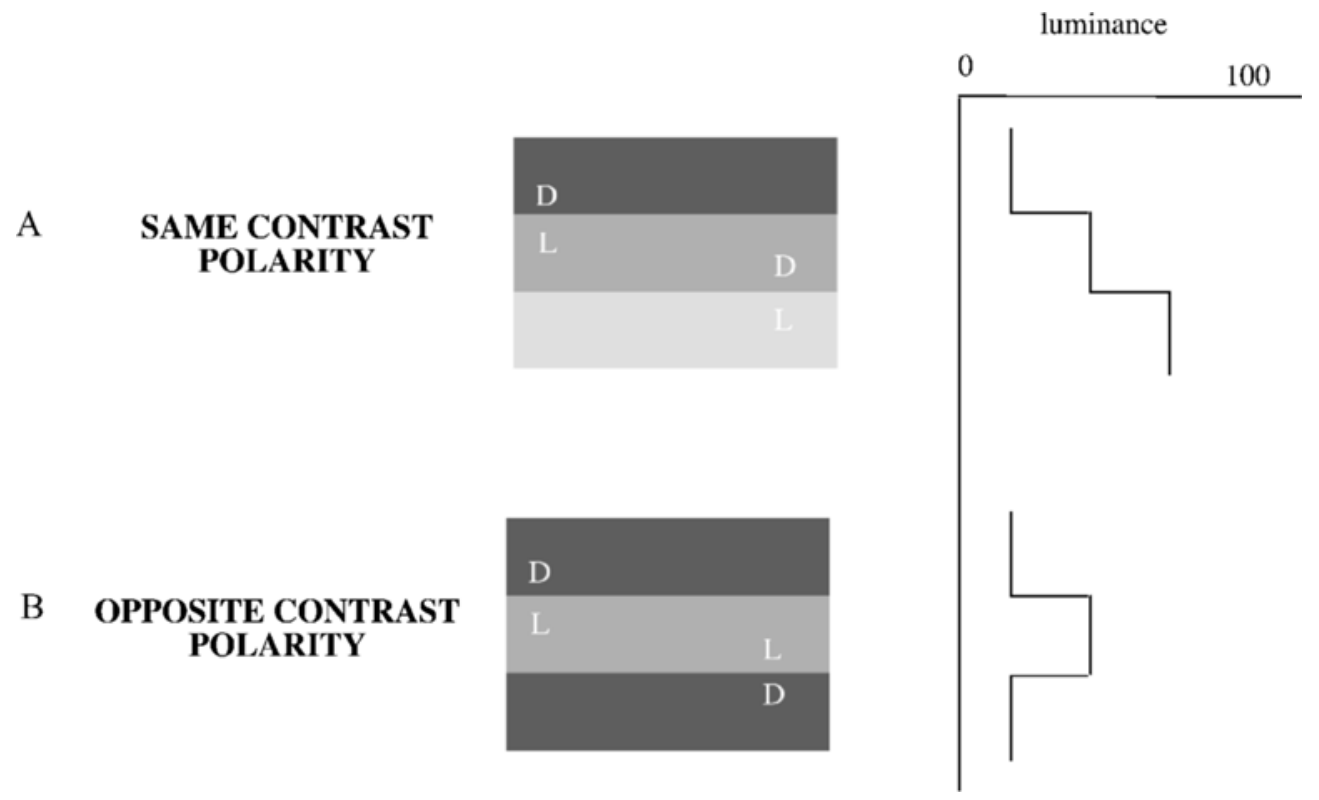

Figure 2. Two edges having the same polarity of contrast and two edges having opposite contrast polarity. Polarity of contrast is not determined, as is usually done, by assessing whether there is an increase or decrease of luminance from the figure to the background, because this would lead to contrast polarity labeling (either same or different), which would depend on what is considered as background. Instead, the contrast polarity of a pair of edges was labeled by considering their luminance profile (as represented on the right) and was labeled as either both $\mathrm{D} / \mathrm{L}$ (or $\mathrm{L} / \mathrm{D}$ ) or one $\mathrm{D} / \mathrm{L}$ and the other $\mathrm{L} / \mathrm{D}$, depending on whether their luminance varies in the same direction (same contrast polarity, panel $\mathrm{A}$ ) or in the opposite direction (opposite contrast polarity, panel B).

edges, is small, and (3) they run parallel for part of their linear extent (they laterally overlap).

The prediction for Condition 1 can be phenomenologically demonstrated because noncollinear edges with opposite polarity are not perceived to join (Figure 3B). The prediction for Condition 2 can be phenomenologically demonstrated because larger vertical distances between noncollinear edges cause the effect to disappear (Figure $3 \mathrm{C}$ ). The prediction for Condition 3 can be phenomenologically proven in two ways: First, a condition of nonoverlapping does not produce distorting effects (Figure 3D); second, if the direction of overlapping is inverted, the direction of distortion also will be inverted (Figure 3E).

The aims of the following experiments were to provide quantitative confirmation, in normal viewing conditions, that the boundary integration affects noncollinear (parallel) edges in different ways, depending on whether they have the same or different contrast polarity, and to explore the role of spatial interactions between two noncollinear edges.

\section{EXPERIMENT 1A}

This experiment was designed to test the predictions that can be drawn from the hypothesis that edges of same contrast polarity tend to align. This was carried out using configurations such as those in Figure 4, similar to that in Figure $1 \mathrm{~B}$, in which either the contrast between neighboring regions or the vertical distance between edges was varied. The outlines on the right side of Figure 4 schematize the edges separating the upper dividing strip from the neighboring regions. In the central zone of Figures $4 \mathrm{~A}$ and 4B, edges of the same contrast polarity laterally overlap for some length before vanishing in opposite directions. We hypothesize that this is a condition for edge conjunction in the direction indicated by the dotted oblique line. In Figure $4 \mathrm{C}$, the condition of equilibrium can be seen, where the two pairs of noncollinear edges do not present lateral overlap in the central region, so that they are interrupted by a region of uniform luminance at the same point.

\section{Method}

Subjects. Four naive subjects and the authors participated in the experiment.

Stimuli. Figures 4A and 4B give two examples of the stimuli employed. Three rectangles $(66 \times 20 \mathrm{~mm})$ were vertically aligned along their shorter sides and separated by a straight strip $(66 \mathrm{~mm}$ long) that can be considered as divided into three regions: black $\left(22 \mathrm{~mm}\right.$ wide, $\left.5.4 \mathrm{~cd} / \mathrm{m}^{2}\right)$ and white $\left(22 \mathrm{~mm}\right.$ long, $\left.93.08 \mathrm{~cd} / \mathrm{m}^{2}\right) \mathrm{re}-$ gions at the extremes and a central region $(22 \mathrm{~mm}$ long) divided into seven rectangles $(3.1 \mathrm{~mm}$ long) of different gray tones. They were ordered so that their luminance progressively increased/decreased in steps of $12 \mathrm{~cd} / \mathrm{m}^{2}$ on average $(12.1,24.3,37.2,49.9,60.8,73.0$, and $84.3 \mathrm{~cd} / \mathrm{m}^{2}$ ). Two strips with opposite directions of variable shading were prepared. Twenty-one different patterns were obtained by combining pairs of luminances taken from the seven luminance values listed above: One value was assigned to the central rectangle, and the other to the isoluminant upper and lower rectan- 
gles. The same rectangle combinations could appear with separating strips, both of which varied in shading from black to white or in the opposite direction; thus, the total number of patterns was 42 . Four control patterns also were used, made up of rectangles of the same luminance (see Figure 4C for an example). The stimuli were computed by the MCDraw program and were presented on an Apple Multiple Scan 15 display (12.4-in. diagonal viewable image size).

Procedure. The method of limits was employed. From each of the 46 basic stimuli, a series of 13 patterns was generated by varying the thickness of the dividing strip. The initial zero value was progressively varied in $0.32-\mathrm{mm}$ steps to obtain a strip width ranging from 0 to $3.8 \mathrm{~mm}$. The 13 width levels were presented in increasing or decreasing order of thickness. The subjects were instructed to observe, after each configuration had been presented, the strips separating the gray rectangles and report whether they saw two rectilinear contours or a deviation from linearity. If the latter occurred, they had to indicate the type of deviation or the direction of convergence. The stimuli were presented in two series in random order; the choice of initial pattern was also random ascending or descending, for each series. Three viewing distances were employed: 120, 180, and $240 \mathrm{~cm}$ in independent conditions. Each subject initiated the experiment with five trials that served for familiarization with the procedure. The stimuli were then observed at the shortest viewing distance, followed by the middle and the longest distance sessions.

\section{Results}

The predicted direction of apparent convergence was consistent with the observers' judgments in $100 \%$ of the cases, since all the observers perceived the configuration in Figure 4A to slant in an opposite direction from that in Figure 4B. This confirmed that the direction of distortion (counterclockwise vs. clockwise) depends on the relative position (either left-right or right-left) of isoluminant regions. For the configuration in Figure $4 C$, the noncollinear edges were not perceived to slant and join, in accordance with the prediction that lateral overlap in the central region between two noncollinear edges is necessary for them to slant and join. The lowest and highest mean values of strip width that gave rise to the illusion of convergence/divergence were calculated for each viewing distance. Table 1 summarizes the results with different viewing distances. Table 2 summarizes the results in relation to the mean luminance difference, calculated for six groups of stimuli. The groups were obtained by assigning the 21 stimuli to six categories according to the difference in luminance $\left(\mathrm{cd} / \mathrm{m}^{2}\right)$ between the central and the lateral rectangles. The groups had decreasing numerosity: from six stimuli (i.e., the configurations in which the rectangles differed in luminance of about $12 \mathrm{~cd} / \mathrm{m}^{2}$ ), to one stimulus (i.e., the configuration showing the greatest difference in luminance; $72.2 \mathrm{~cd} / \mathrm{m}^{2}$ ).

The data in Table 1 show that the illusory slant was perceived at the minimum separation $\left(D_{\min }\right)$ of about 1 arcmin. The mean widest extent $\left(D_{\max }\right)$ within which the distorting effect occurred was almost 4 arcmin at the shortest viewing distance and smaller than this at longer distances. An analysis of variance carried out on the minimum and maximum values gave the following results. The $D_{\min }$ and $D_{\max }$ values decreased when the observing distance increased $\left[D_{\min }, F(2,10)=11.72, p<\right.$ $\left..005 ; D_{\max }, F(2,10)=7.56, p<.01\right]$. Post hoc comparisons (Newman-Keuls) demonstrated that significant differences existed between results obtained at the shortest distance and those obtained at longer distances. The difference in luminance produced differences in minimum $D_{\min }$ values $[F(5,25)=8.67, p<.001]$ and $D_{\max }$ values $[F(5,25)=16.22, p<.001]$.

\section{Discussion}

The results are in line with the prediction that noncollinear parallel edges having the same contrast polarity tend to align; the tendency has perceivable effects with a $D_{\text {max }}$ of less than 4 arcmin. $D_{\text {max }}$ decreases (up to $30 \%$ ) as viewing distance is increased. A second result is an increase in $D_{\min }$ and $D_{\max }$ magnitude with increases in luminance difference (i.e., contrast). However, Table 2 shows that $D_{\max }$ and $D_{\min }$ do not increase linearly with contrast and that the $D_{\max }-D_{\min }$ difference is almost fixed (about 2 arcmin). This is odd, because the contrast covaries with lateral overlap, which, according to our predictions, affects the distortion. In the following experiment, $1 \mathrm{~B}$, we attempted to disentangle the role of contrast from that of lateral overlap by measuring the lateral overlap threshold in two contrast conditions. If lateral overlapping is important, rather than contrast per se, thresholds should not vary in the two contrast conditions.

\section{EXPERIMENT 1B}

\section{Method}

Subjects. Three naive subjects, 1 experienced (A.G.), and the authors participated in the experiment.

Stimuli. Figure 4A gives an example of the stimuli employed. A central rectangle was separated from two external ones having the same size $(27.3 \times 8.4 \mathrm{~mm})$, but either lower or higher luminance, by a straight strip $(27.3 \times 0.54 \mathrm{~mm})$. The strip luminance varied in shading from black to white in a central region according to the constraint that overlapping regions of equal extent had to be obtained in patterns of different contrast. We obtained two sets of pattern, one with high contrast $\left(38.2\right.$ and $\left.9.53 \mathrm{~cd} / \mathrm{m}^{2}\right)$, the other with low contrast $\left(23.2\right.$ and $\left.9.53 \mathrm{~cd} / \mathrm{m}^{2}\right)$, and in each the extent of overlapping varied according to five levels $(0,7,14,21$, and by 28 arcmin).

Procedure. In each trial, two successive stimuli were presented, one with no lateral overlap (standard stimulus, hereafter SS) and the other with one of five levels of lateral overlap (comparison stimulus, hereafter SC). The order of presentation of SS and SC was randomly varied from trial to trial. The stimulus duration was $300 \mathrm{msec}$, and the interstimulus interval was $1 \mathrm{sec}$, whereas the viewing distance was $120 \mathrm{~cm}$. A two-alternative temporal choice was used in which the observer had to indicate in which of the two intervals the strips separating the two rectangles were perceived as a continuous contour deviating from linearity, rather than as two noncollinear contour segments. The percentage of times that the interval containing SC was chosen was calculated for each width of overlap of the edges, independently for the two contrast levels. Thresholds, defined as the width of the central region at which the observers chose the interval containing the SC $75 \%$ of the time, were calculated by Probit analysis (Finney, 1971). The stimuli were presented on a Multiscan Computer Display (420GS).

\section{Results}

Individual thresholds in high-contrast condition s were 11.63 (R.S.), 1.2 (A.G.), 5.7 (F.T.), 5 (C.C.), 10 (R.C.), and 10 (S.L.) arcmin. Individual threshold in the low-contrast condition were 7.65 (R.S.), 3.0 (A.G.), 3.8 (F.T), 7.8 
A

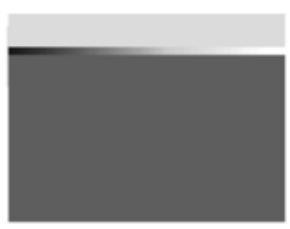

B

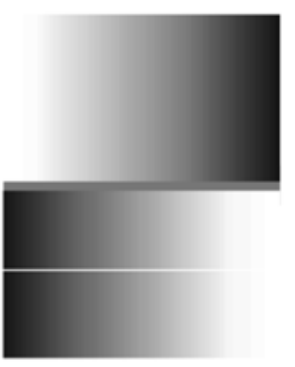

C

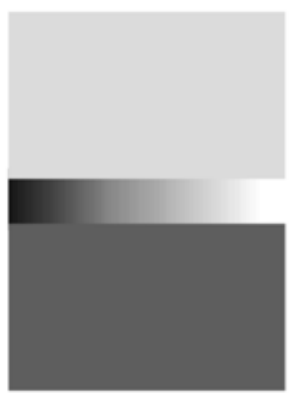

D
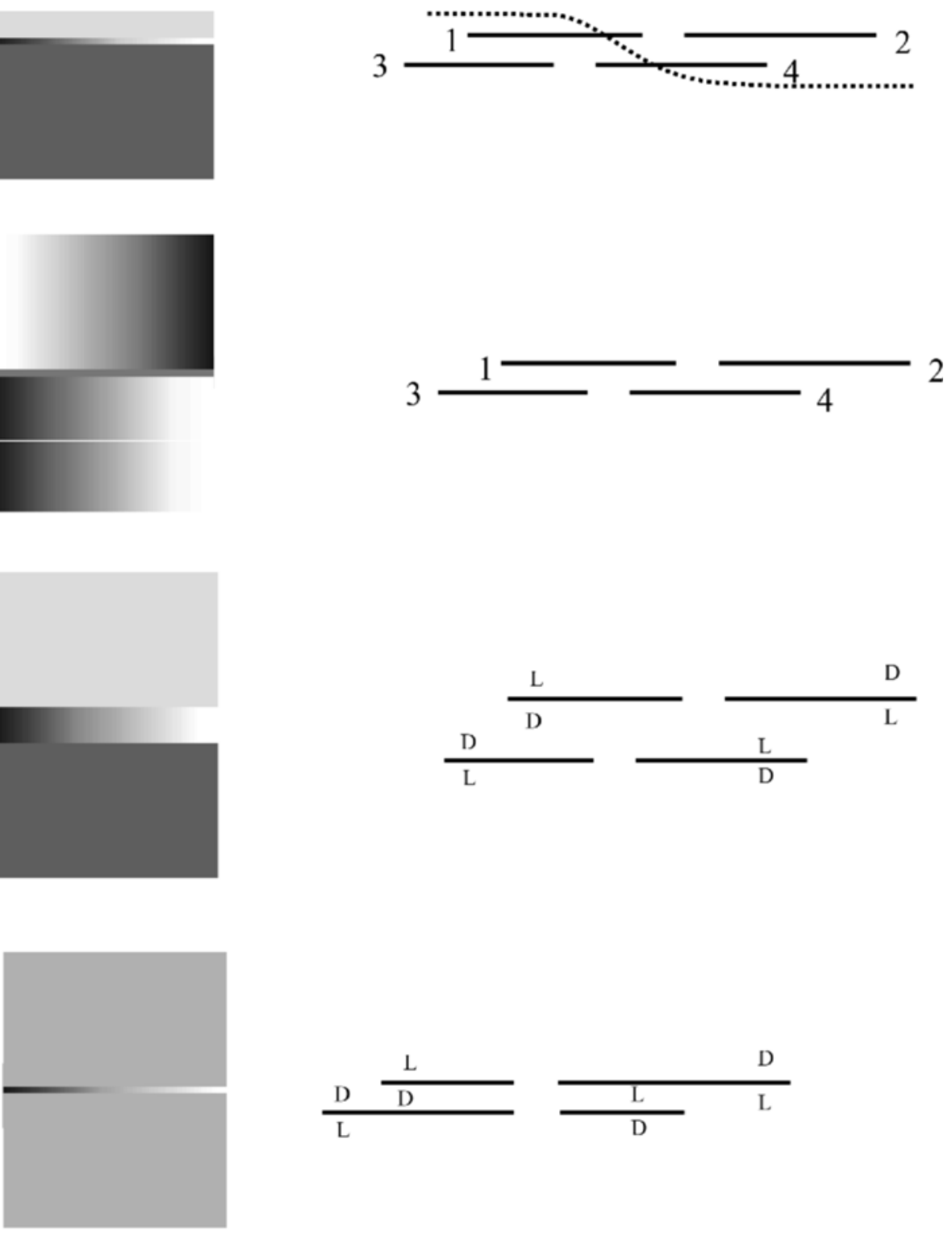

E
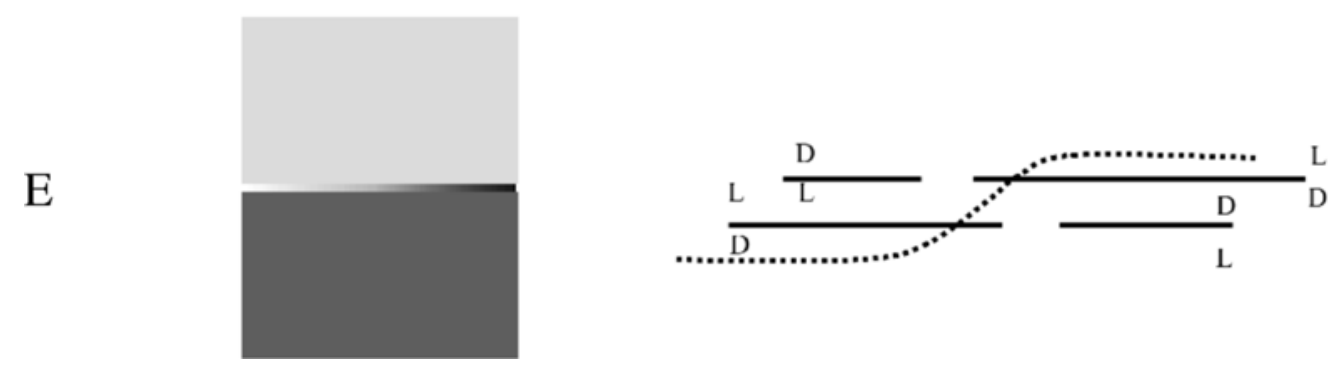

Figure 3. Figure caption appears on the following page. 
Figure 3. The outline on the right of panel A illustrates that, in the central region of the corresponding edge configuration on the left, Collinear Edges 1 and 2 have opposite contrast polarity, Collinear Edges 3 and 4 have opposite contrast polarity, Noncollinear Edges 3 and 2 have same contrast polarity, and Noncollinear Edges 1 and 4 have same contrast polarity and run parallel in the central region (laterally overlap). Panel $B$ shows that noncollinear edges ( 1 and 4$)$ with opposite polarity are not perceived to join. Panel $\mathrm{C}$ phenomenologically demonstrates that large vertical distances between noncollinear edges cause the effect in Figure $1 \mathrm{~B}$ to disappear. Panel $D$ shows that a condition of nonoverlapping does not produce distorting effects. Panel $E$ phenomenologically illustrates that if, in comparison with panel $\mathrm{A}$, the direction of overlapping is inverted, so is the direction of distortion.

(C.C.), 14.5 (R.C.), and 7 (S.L.). Mean thresholds were equal to 7.25 and 7.29 arcmin in the high-contrast and in the low-contrast conditions, respectively. This result, that the threshold was the same in the two contrast con- ditions, demonstrates that what was important in generating the distortion was the presence of the central variable shading region, rather than the difference in luminance between regions. The mean extent of the shading region for the distortion to be perceived was $7.27 \mathrm{arcmin}$ $(S D=3.83)$.

\section{Discussion}

The results of Experiments $1 \mathrm{~A}$ and $1 \mathrm{~B}$ give no suggestion regarding the source of the misperception we called tendency of edges of same contrast polarity to join. One possible explanation follows from previous psychophysical research documenting the misperception of distances separating segments or edges (Badcock \& Westheimer, 1985a, 1985b; Greene \& Brown, 1995; Rentschler, Hilz, \& Grimm, 1975). Both attraction and repulsion effects have been described - that is, a test line appeared that was located, with respect to the flank line,
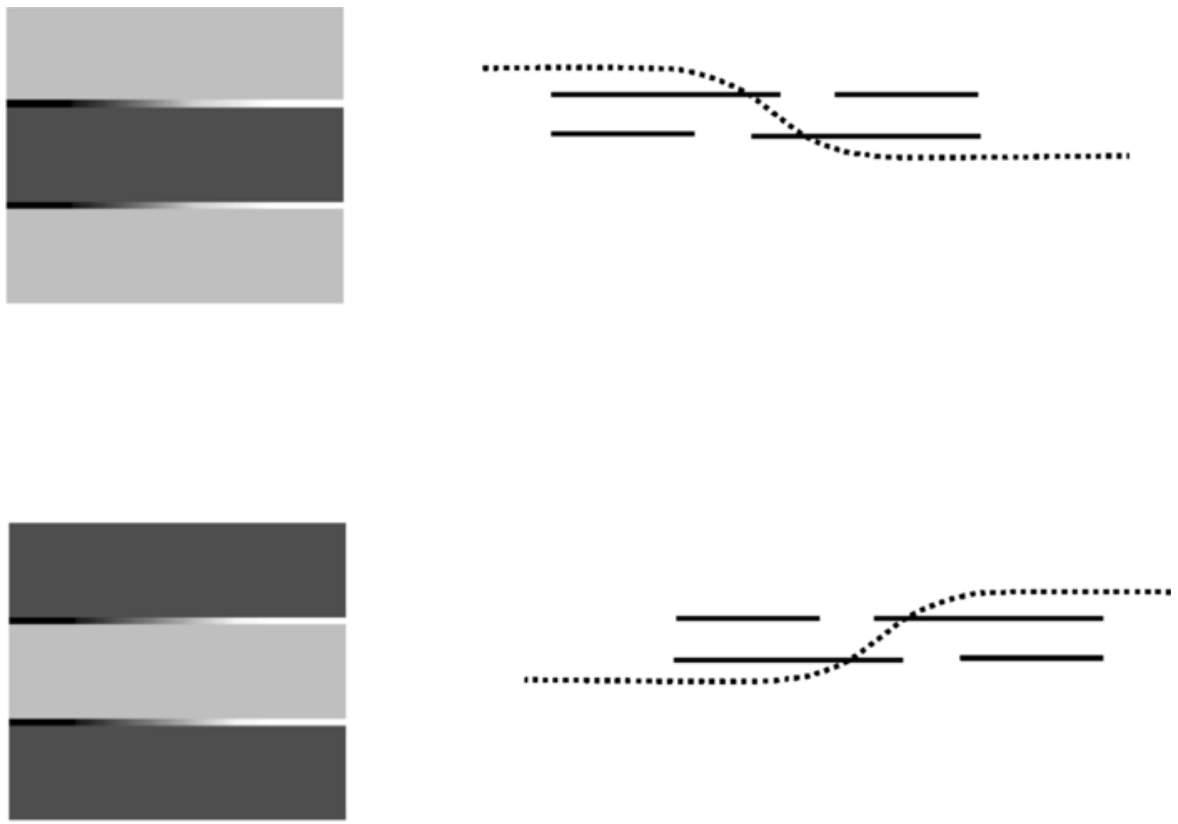

$\mathrm{C}$
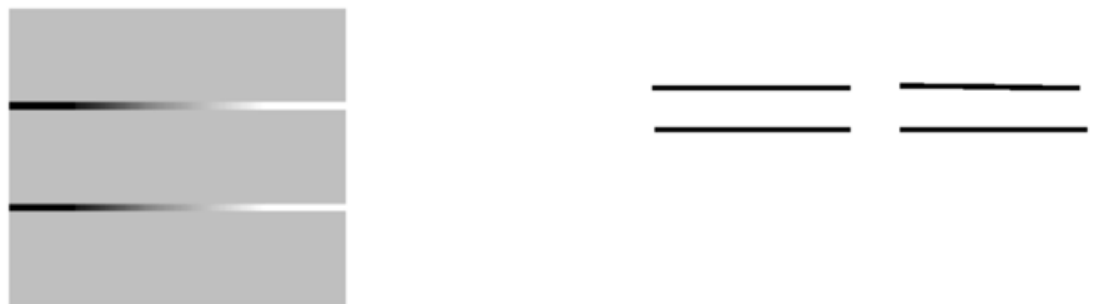

Figure 4. Examples of the stimuli employed in Experiment 1A. Patterns in panels A and B reproduce the experimental stimuli-that is, the outlines on the right indicate the edges, and the dotted lines the predicted direction of edge completion. Panel $\mathrm{C}$ reproduces one of the control stimuli (where the dividing strips are not expected to distort). 
Table 1

Mean Values (in Arcmin) of the Minima $\left(D_{\min }\right)$ and Maxima $\left(D_{\max }\right)$ Values of Contour Thickness Giving Rise to the Illusory Convergence/Divergence of the Dividing Strips as a Function of Viewing Distance in Experiment 1

Viewing Distance

\begin{tabular}{cccc}
$(\mathrm{cm})$ & $D_{\min }$ & $D_{\max }$ & $D_{\max }-D_{\min }$ \\
120 & 1.25 & 3.91 & 2.66 \\
180 & 0.81 & 3.17 & 2.36 \\
240 & 0.75 & 2.96 & 2.21 \\
\hline
\end{tabular}

at a distance shorter than the true value and farther away, respectively. These effects-attraction, in particulardecreased in magnitude as the distance increased, with the highest magnitudes registered within a range of 3-4 arcmin of visual angle. This is an important analogy with our results, since this distance corresponds to the $D_{\max }$ value at which we observe apparent tilt of dividing line, although in a direction not consistent with the polarity contrast effects found by Badcock and Westheimer (1985b).

In order to assess whether the effects we observed in the previous experiment could be ascribed to the class of attraction/repulsion effects, we carried out a further experiment with two main goals. The first was to investigate whether interaction between the edges had one direction or two, opposite one to another. In a preliminary inquiry, no repulsion effects were registered, but this may have been due to the characteristics of the pattern. Second, our aim was to check whether the direction of interaction was dependent on the contrast polarity, although previous research had not found a precise relation between direction (repulsion vs. attraction) of effect and the contrast polarity. As was discussed in the introduction, it may be possible that in the configurations we used, noncollinear edges of both the same and opposite contrast polarity interact, but in a different way. We hypothesized that edges of the same contrast polarity would tend to attract and would be perceived to be closer than they really were, whereas those of opposite polarity contrast would tend to repel and would be perceived to be farther than they really were.

\section{EXPERIMENT 2}

To address these issues, it was necessary to use a different stimulus configuration. However, this was designed in such a way that the spatial arrangement between edges corresponded to that in the stimuli used in Experiment $1 \mathrm{~A}$. Figure 5 illustrates the pattern producing this arrangement. In Figure 5A, the black and white rectangles are noncollinear, laterally displaced and embedded in a region of uniform luminance. As the outline on the right shows, the edges between the rectangles and the region of uniform luminance are also noncollinear and laterally overlapping for part of their length. We saw in Experiment $1 \mathrm{~A}$ that this spatial arrangement is crucial to perception of the edges as slanted, and it is maintained in Experiment 2. Again, we predicted that the direction of distortion (counterclockwise vs. clockwise) should depend on the relative position (left-right) of the points where the edges are interrupted by the uniform luminance background. In the configurations in Figure 5A, one rectangle is black and one white, forming edges of the same contrast polarity. In the configuration in Figure $5 \mathrm{~B}$, the two rectangles have the same luminance, so that the edges they form have opposite contrast polarity. On the basis of the attraction and repulsion hypothesis (illustrated in the outline by dotted lines), the two edges of the same contrast polarity should be perceived as slanting and aligning, whereas the two edges with the opposite contrast polarity should be perceived as slanting and misaligning. This concept is better illustrated in the right-hand configurations on the right side in Figures $5 \mathrm{~A}$ and $5 \mathrm{~B}$, which illustrate how edges appear to rotate in a direction that makes them perceptually align (Figure 5A) or in a direction in which they perceptually misalign (Figure 5B).

The stimuli used in Experiment 2 are shown on the left side of Figure 6 and consist in a repetition of the basic configurations of Figure 5. These are made up of rows of alternating light and dark gray squares; in each row, the squares are slightly displaced laterally so that they laterally overlap with noncollinear squares of the row above or below. Three conditions are created, depending on whether overlapping occurs between squares of (1) a different color, (2) the same color, or (3) both the same and a different color. The predictions generated by the attraction-repulsion hypothesis (see the enlargements) are schematized by arrows indicating the direction of shift the edges should undertake.

Condition 1 is illustrated in Figure 6B. The enlargements show that, in this configuration, edges with the same contrast polarity laterally overlap, whereas those with opposite polarity do not. The direction of perceived tilt (illustrated by dotted lines in panel B3) is consistent with the hypothesis that only edge pairs with appropriate arrangement (those with the same contrast polarity, since they laterally overlap for part of their lengths) are perceived as tilted, in a direction predicted by the hypothesis that edges of same contrast polarity tend to align.

Table 2

Mean Values (in Arcmin) of $D_{\min }$ and $D_{\max }$ Giving Rise to the Illusory Convergence/Divergence of the Dividing Strips as a Function of the Difference in Region Luminance in Experiment 1

\begin{tabular}{cccc}
\hline $\begin{array}{c}\text { Mean Difference } \\
\text { in Luminance } \\
\left(\mathrm{cd} / \mathrm{m}^{2}\right)\end{array}$ & $D_{\min }$ & $D_{\max }$ & $D_{\max }-D_{\min }$ \\
\hline 12.0 & 0.73 & 2.73 & 2.00 \\
24.2 & 0.76 & 3.08 & 2.32 \\
35.8 & 0.88 & 3.24 & 2.36 \\
48.1 & 0.93 & 3.48 & 2.55 \\
60.4 & 1.11 & 3.60 & 2.49 \\
72.2 & 1.18 & 3.87 & 2.69 \\
\hline
\end{tabular}




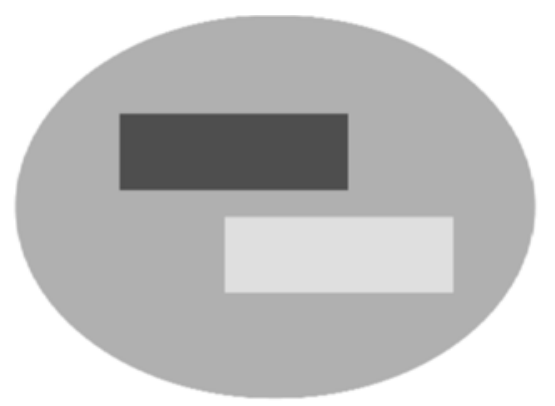

A

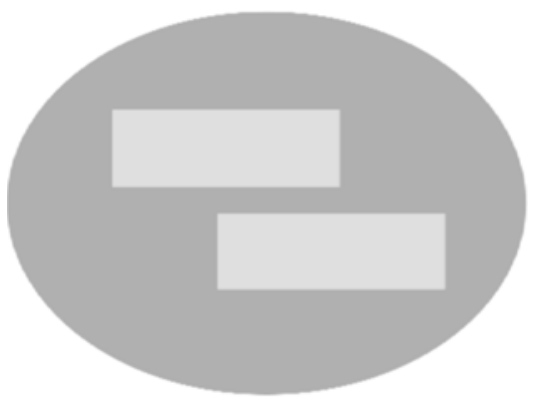

B
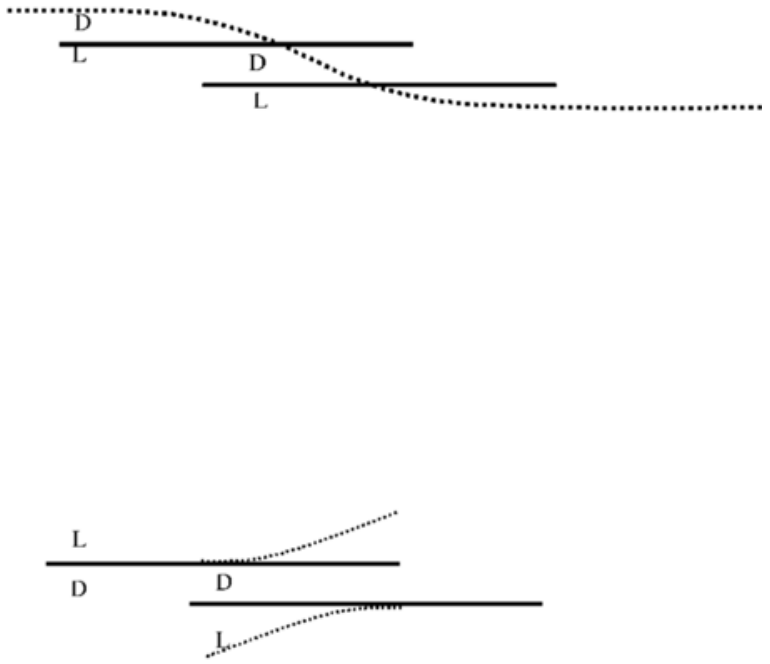

Figure 5. In panel $A$, one rectangle is black, and one is white, forming edges of the same contrast polarity. In the configuration in panel $B$, the two rectangles have the same luminance, so that the edges they form have opposite contrast polarity. On the basis of the attraction and repulsion hypothesis (illustrated in the outline by dotted lines), the two edges of the same contrast polarity should be perceived as slanting and aligning, whereas the two edges with the opposite contrast polarity should be perceived as slanting and diverging (misaligning).

Condition 2 is illustrated in Figure 6C. The enlargements show that, in this configuration, edges with opposite contrast polarity laterally overlap, whereas those with the same polarity do not. The direction of perceived tilt (illustrated by dotted lines in panel C3) is consistent with the hypothesis that only pairs of edges in the appropriate spatial arrangement (those with opposite contrast polarity, since these laterally overlap for part of their lengths) are perceived as tilted, in a direction predicted by the hypothesis that edges of opposite contrast polarity tend to misalign.

Condition 3 is illustrated in Figure 6D. The enlargements show that, in this configuration, pairs of edges with both the same and opposite contrast polarity overlap. The direction of overall perceived tilt (illustrated by dotted lines in panel D3) is consistent with the hypothesis that when there is partial lateral overlap between them, edges with the same contrast polarity tend to align, whereas edges with opposite contrast polarity tend to misalign.

Moreover, another condition was presented, illustrated in Figure 6A. To understand its relevance, it should be recalled that one of our main predictions was that the direction of distortion (counterclockwise or clockwise) should depend on the relative position (left-right) of the point at which the edges are interrupted by a region of uniform luminance. Figure $6 \mathrm{~A}$ demonstrates phenomenologically that this prediction is correct. In this configuration, the rows of alternating dark and light squares are embedded in a white background. In this way, all pairs of edges (those between two light squares, those between two dark squares, and those between one dark and one light square) are of opposite contrast polarity. However, as the dotted lines in Figure 6A3 show, the relative position of these edges alternated, so that in one of two contiguous edge pairs the edge above is shifted rightward from that below, whereas in the other it is shifted leftward. For this reason, although all these pairs of edges are perceived to misalign, since they have opposite contrast polarity, the direction of alignment alternates, so that, overall, there is no perceived slant. This configuration was used as a control in Experiment 2.

\section{Method}

Subjects. Ten subjects, all undergraduates, participated in the experiment.

Stimuli. Examples of the stimuli are shown in Figure 6; they consisted of rows of alternate light and dark squares $(8 \times 8 \mathrm{~mm})$ aligned along their horizontal axis and arranged in rows. Square separation was equal to $6.0 \mathrm{~mm}$ in Conditions 1 and 2 and to $4.8 \mathrm{~mm}$ in Condition 3 and the control condition. The rows were constructed in the 
A1

A2

A

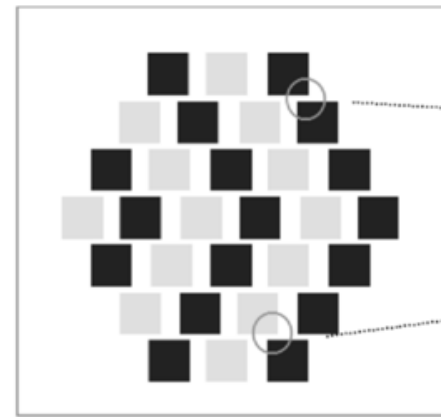

B1

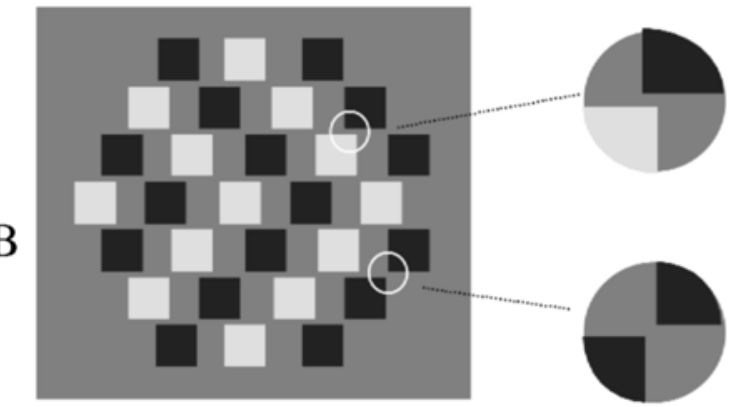

C1

C

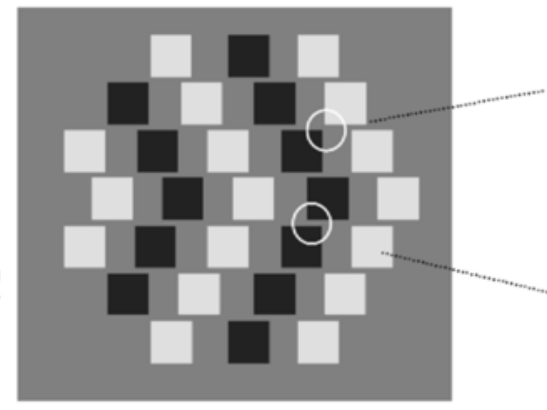

D1

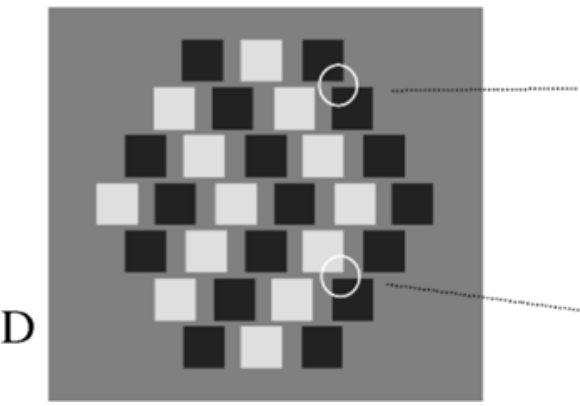

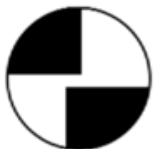

C2
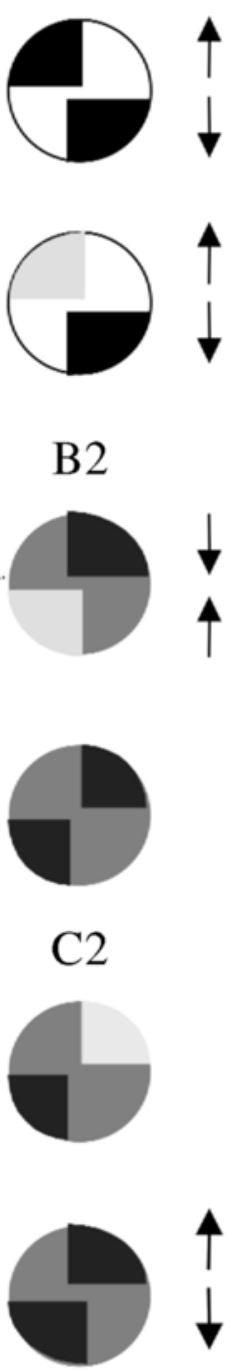

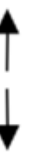

B2

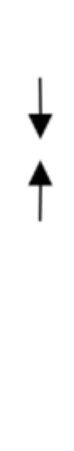

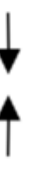
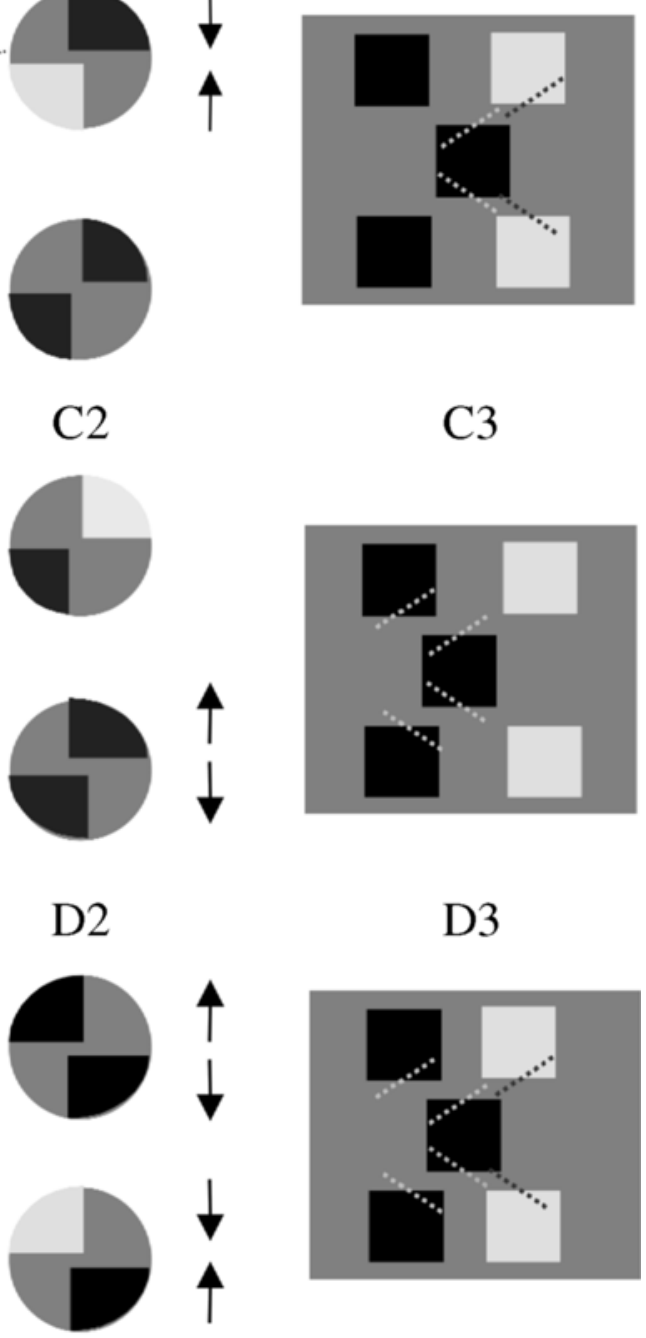

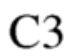

D3

A3

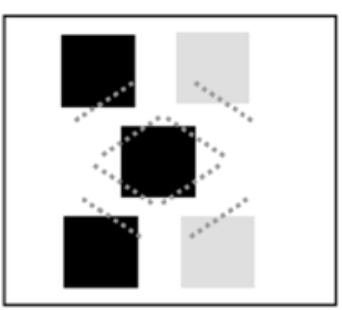

B3

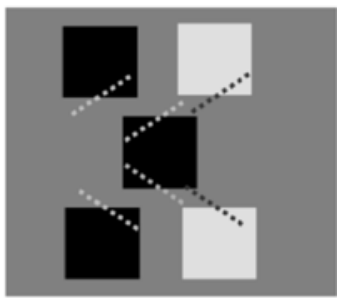

Figure 6. Panels A and D reproduce the same arrangement of squares on different backgrounds. In panel A, the background has the highest luminance of the depicted regions; in this condition, the overlapping edges have opposite contrast polarity. In panels $B, C$, and $D$, the background luminance is intermediate between those of the light and the dark squares. As panels B2, C2, and D2 show, laterally overlapping edges have either the same or opposite contrast polarities. Converging and diverging arrows symbolize the effects of attraction and repulsion, respectively, of overlapping edges. The tilted dotted lines in panels B3, C3, and D3 illustrate the expected directions of tilt of the overlapping edges. The luminances of panels A, B, C, and $D$ are representative of those used in the stimuli but do not reproduce them exactly (see text for details). 
following way. Each row was displaced with respect to that above or below so as to produce the three main conf igurations used in the experiment. The amount of overlap was $2.9 \mathrm{~mm}$ in Conditions 1 and 2 and $1.6 \mathrm{~mm}$ in Condition 3 and the control condition. The configurations in Figures $6 \mathrm{~A}-6 \mathrm{D}$ will be referred to as basic configurations henceforth. The background luminance, $34.04 \mathrm{~cd} / \mathrm{m}^{2}$, was the same in all basic configurations. Each was presented in 2 contrast conditions. In the high-contrast condition, the luminance of the dark square was $6.05 \mathrm{~cd} / \mathrm{m}^{2}$ and that of a light square was $61.3 \mathrm{~cd} / \mathrm{m}^{2}$. In the lowcontrast condition, the luminance values were 15.12 and $52.04 \mathrm{~cd} / \mathrm{m}^{2}$, respectively. Three high-contrast and three low-contrast basic configurations were obtained. For each of the 6 conditions, two square arrangements were prepared in which the illusory tilt was in opposite directions. In addition to the 12 conditions described above, the following 3 control conditions were used. One is illustrated in Figure $6 \mathrm{~A}$ and consisted of rows of squares of two different grays alternating on a light background. The second consisted of rows of squares of the same gray on a lighter background. The third consisted of rows of squares of the same gray on a darker background. In these three configurations, all the edges have opposite contrast polarity and are perceived to misalign in a direction that alternates from clockwise to counterclockwise, as is illustrated in Figure 6A3. The stimuli were computed by the MCDraw program and were presented on an Apple Multiple Scan 15 display (12.4-in. diagonal viewable image size).

Procedure. The method of limits was employed. From the 15 basic conditions, a series of patterns was generated by varying the distance between rows of squares. The initial zero value was progressively increased by $0.32-\mathrm{mm}$ steps up to a distance of $3.8 \mathrm{~mm}$. The 13 patterns were presented to the subjects in order of either increasing or decreasing vertical distance between the rectangles; the observers were instructed to inspect each configuration and to report whether or not they saw a convergence of the rows. In the case of a positive response, they were also asked to indicate the direction of convergence. Two viewing distances were employed: 120 and $180 \mathrm{~cm}$, balanced among subjects.

\section{Results}

The subjects' judgments of convergence/divergence were in full agreement with the hypothesis that edges of the same contrast polarity tend to align and that edges of opposite contrast polarity tend to misalign. With null distance between the rows, no misperception occurred, and the dividing horizontal lines were always perceived as horizontal. With increasing distance, the rows appeared to converge (or diverge) toward a direction consistent with the hypothesis that edges with the same contrast polarity tend to align and those with opposite polarity tend to misalign. Four subjects observed an inversion of the phenomenon with large distance separations (over $7-8$ arcmin of visual angle; the proportion is $8.7 \%$ of the total of 240 trials), but these impressions were not systematic and cannot be related to a particular variable. For the control pattern, none of the subjects perceived the effect of convergence or divergence of rows. The mean values of distance between squares giving rise to an illusory convergence of the dividing lines are reported in Table 3.

When the distance between the rows of squares had mean $D_{\max }$ values higher than about 3.27 arcmin at $120 \mathrm{~cm}$ and of $2.76 \mathrm{arcmin}$ at $180 \mathrm{~cm}$ viewing distance, the illusory convergence disappeared. As in Experiment 1, both the effect of viewing distance on $D_{\max }[F(1,9)=$
Table 3

Mean Values (in Arcmin) of $D_{\min }$ and $D_{\max }$ Giving Rise to the Illusory Convergence/Divergence of the Dividing Strips in Relation to Viewing Distance in Experiment 2

\begin{tabular}{cccc}
\hline $\begin{array}{c}\text { Viewing Distance } \\
(\mathrm{cm})\end{array}$ & $D_{\min }$ & $D_{\max }$ & $D_{\max }-D_{\min }$ \\
\hline 120 & 0.98 & 3.27 & 2.29 \\
180 & 0.77 & 2.76 & 1.99 \\
\hline
\end{tabular}

$17.59, p<.005]$ and $D_{\min }[F(1,9)=12.31, p<.01]$ were statistically significant. The three conditions of overlapping produced statistical difference in both $D_{\min }$ and $D_{\max }$ values $[F(2,18)=3.65, p<.05$, and $F(2,18)=6.11$, $p<.01$, respectively]. Pairwise comparisons (NewmanKeuls) demonstrated that the $D_{\max }$ with edges of opposite contrast polarity was higher than those in the other two conditions. The differences in contrast significantly affected $D_{\max }$ values $[F(1,9)=18.34, p<.005]$. These results can be summed up as follows. Viewing distance affects both minimum and maximum values, perhaps because as viewing distance increases, the amount of overlap, expressed in visual angle, decreases. Interaction attributable to edges of opposite contrast polarity occurs within a larger range of action with respect to the interaction between edges of the same polarity. Low contrasted shapes interact at longer distances than higher contrasted shapes. With distances larger than the $D_{\max }$ values reported in Table 3, the illusory effects are not consistent. In some cases, a reversing of the illusion is perceived.

\section{Discussion}

This experiment confirms the results of Experiment $1 \mathrm{~A}$ with regard to the extension of the region within which two edges interact; this extension has a mean width of $2.14 \mathrm{arcmin}$. The contrast has little influence and enhances the effect as it is reduced, but this effect is weak (14-arcsec difference), a result in line with the findings of Bex and Edgar (1996) that apparent locations of sharp edges are unaffected by contrast. The second important result is consistent with the prediction that interaction between edges generates a strong misperception of their alignment in a direction predictable on the basis of contrast polarity, whether the same or different. Moreover, the two opposing effects - that is, the tendency of edges with the same contrast polarity to align and of those with different contrast polarity to misalign-occurs within ranges very similar in magnitude. Finally, the attraction and repulsion forces do not sum, since their combined action (as in the configuration of Figure 6D) does not increase the magnitude of the distortion effects.

\section{EXPERIMENT 2A}

In the stimulus configurations in Experiment 2, one confounding factor was that squares of the same luminance may group together; this provided a global luminance cue that could be used by the subjects when judg- 
Table 4

Mean Values (in Arcmin) of $D_{\min }$ and $D_{\max }$ Distances Between the Edges in Stimuli of Experiment 2 for Illusory Effects to be Perceived in Relation to the Contrast Sign of the Interacting Edges

\begin{tabular}{cccc}
\hline Edges Contrast Sign & $D_{\min }$ & $D_{\max }$ & $D_{\max }-D_{\min }$ \\
\hline Positive & 0.90 & 2.92 & 2.02 \\
Negative & 0.84 & 3.13 & 2.29 \\
Neutral & 0.87 & 2.99 & 2.12 \\
\hline
\end{tabular}

Note-Positive, same contrast polarity as illustrated in Figure 6B; negative, opposite contrast polarity as illustrated in Figure 6C; neutral, both types of edge interact as illustrated in Figure 6D.

ing whether or not they perceived a converging of rows. In the stimuli of Experiment 2A, this cue was eliminated.

\section{Method}

Subjects. The authors and two naive subjects participated in the experiment.

Stimuli. The stimuli were a simplified version of those in Experiment 2 and consisted of five rows of rectangles $(6.2 \times 3.8 \mathrm{~mm})$ separated by a given distance. The central row had five rectangles, whereas in those above and below, the number of rectangles progressively increased and decreased to form a symmetrical horizontal pattern. The interrectangle distances $(4.6 \mathrm{~mm})$ were chosen so that each rectangle in a row partially overlapped laterally with those in the counterphase rows above and below. In this way, as in Condition 3 of Experiment 2, both pairs of edges with the same and opposite contrast polarity overlapped $(0.81 \mathrm{~mm})$. By varying the relative position of pairs of edges with the same and different contrast polarity, we varied, in two sets of stimuli, the perceived direction of convergence (at the appropriate edge separation). Differently from Experiment 2, rectangles luminance varied randomly in the range of $6.4-16.74 \mathrm{~cd} / \mathrm{m}^{2}$ (for dark rectangles) and $45.8-92.2 \mathrm{~cd} / \mathrm{m}^{2}$ (for light rectangles). Background luminance was $27.8 \mathrm{~cd} / \mathrm{m}^{2}$. The control stimuli were identical to the experimental ones, with the exception that the rectangle's luminance varied randomly in the $6.4-92.2 \mathrm{~cd} / \mathrm{m}^{2}$ range, so that the contrast polarity rules were not respected. Row distance varied from 0 to $2.7 \mathrm{~mm}$ in steps of $0.27 \mathrm{~mm}$ to form a total of 11 stimuli for both experimental and control patterns. In the experimental session of 176 trials, eight repetitions of each of 11 configurations of both experimental and control stimuli were randomly presented, and in each repetition the luminance of the rectangles was randomly varied over the appropriate range to avoid configurational cues. In four of the eight repetitions of the experimental stimuli, the illusory convergence, when perceived, was rightward, and in four it was leftward. The stimuli were presented on a Multiscan Computer Display (420GS).

Procedure. In each trial, each of the 176 patterns was presented in random order for $200 \mathrm{msec}$ at a $120-\mathrm{cm}$ viewing distance. The observers' forced choice was to indicate whether they perceived the rows as converging toward the right or the left.

\section{Results and Discussion}

The results are shown in Figure 7, together with two examples of the stimuli; note that with short interrow distance, the stimuli appear to converge leftward. The proportion of either right or left responses to the stimuli that could generate rightward or leftward convergence, respectively, was calculated. A chi-square test carried out on the two distributions of data gave no significant difference, so the pooled results are plotted in Figure 7. In the figure, the proportion of responses in agreement with the attraction/repulsion hypothesis is shown as a function of interedge distance (in arcmin). The dotted line indicates $75 \%$ of responses in agreement with the hypothesis. Clearly, the percentage of judgments above this threshold level occurred in the range of 0.7-3.7 arcmin. This range is very similar $(0.98-3.27$ arcmin) to that in Experiment 2, demonstrating that the convergence judgments were not biased by global configurational cues. The proportion of right responses to control stimuli was 49 .

\section{EXPERIMENT 3}

From the results of Experiment 2, it appears that the distortion effect may depend on the tendency for attraction and repulsion. However, there are different effects of attraction and repulsion, perhaps resulting from different mechanisms. These consist either of local shifts or of deformations. For example, iron balls move toward an attracting magnet pole with speeds and directions that can differ if the start points are not equidistant from the source of attraction. But very different paths of motions are observed if the balls are fixed to a rigid frame so that they cannot move independently. The balls and the frame will react as a system to the attraction pole, and the path of motion (combination of translation and rotation) will be very different from the paths of independently moving balls. Figure 8 gives a graphic representation of the outcomes of attraction/repulsion interactions when applied either to points free to move or to whole edges. The figure simulates what can occur in these two cases in a region within which two edges laterally overlap.

Point-to-point interaction. This is schematized in Figures $8 \mathrm{~A}$ and $8 \mathrm{~B}$. The points along the part of the edges that laterally overlap attract (or repel) one another. The result of these local shifts, constant in magnitude and direction, is a regular deformation of the overlapping part of both edges in the form of an outer trapezoid (due to attraction; Figure $8 \mathrm{~A}$ ) or an inner trapezoid (Figure 8B). The consequent deformation of this part of the contour can sum with other local deformations and perceptually emerge as an overall contour slant. It has already been shown that this point-to-point attraction/repulsion is unlikely to occur, since it is the real or the illusory contours that spatially interact, not the single points (Greene \& Brown, 1997). Nevertheless, there are other local interactions between opposed line terminators that can give rise to important illusory contours (He \& Ooi, 1998), and this end-to-end interaction can account for perceived deformations.

\section{Table 5}

Mean Values (in Arcmin) of $D_{\min }$ and $D_{\max }$ for Illusory Effects to be Perceived in Relation to the Difference in Luminance (High vs. Low) Between the Squares in the Configuration Stimuli of Experiment 2

\begin{tabular}{cccc}
\hline Luminance Difference & $D_{\min }$ & $D_{\max }$ & $D_{\max }-D_{\min }$ \\
\hline High & 0.86 & 2.89 & 2.03 \\
Low & 0.84 & 3.13 & 2.29 \\
\hline
\end{tabular}




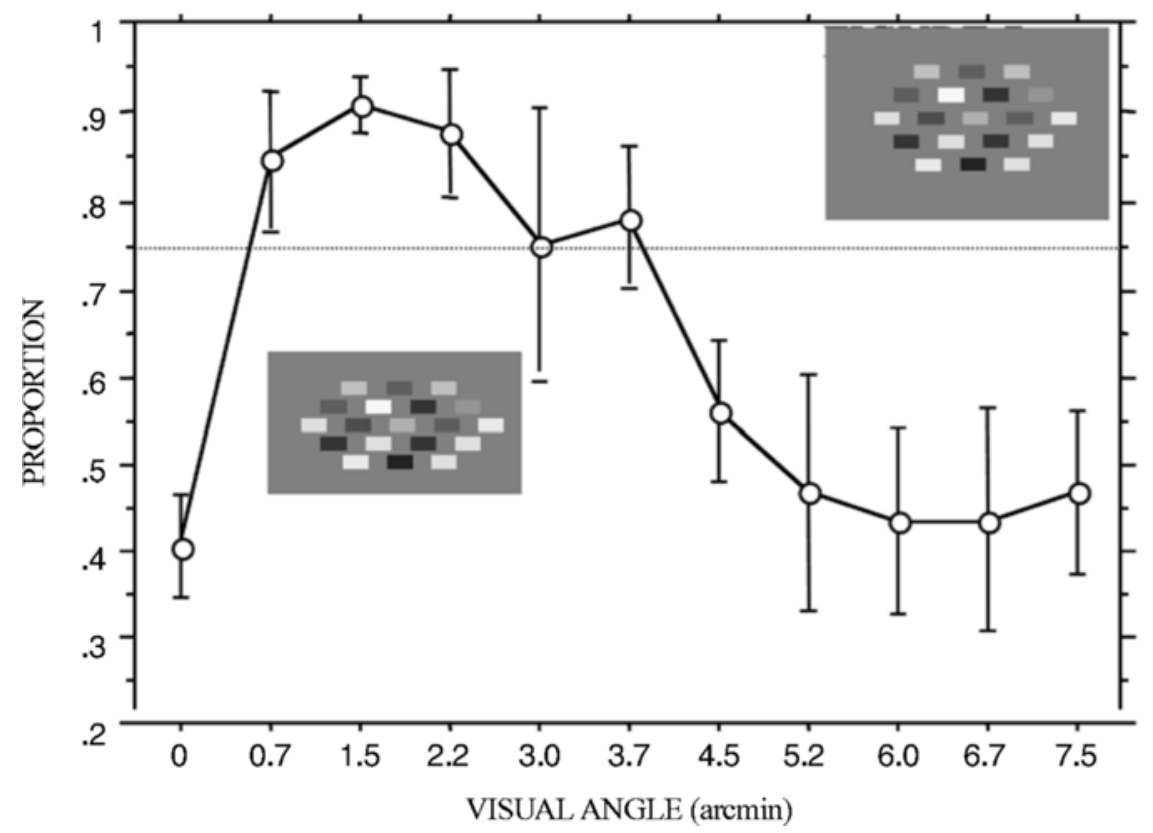

Figure 7. The results of Experiment 2B, with the proportion of reported convergence in either direction (left or right) but consistent with the polarity of contrast hy pothesis, are shown as a function of interrow distance. The dotted line indicates $75 \%$ of responses consistent with the polarity of contrast hy pothesis. The 1.5, 2.2,3.0, and 3.7 data points lie above the threshold level, suggesting that within this range of separation, the direction of perceived convergence is as predicted by the polarity of contrast hypothesis.

Alignment/misalignment (edges) interaction. In Figures $8 \mathrm{C}$ and $8 \mathrm{D}$, the outcomes are illustrated as gray areas of attraction/repulsion forces acting on rigid objects represented by dotted rectangular perimeters. The attraction between the two edges, as is illustrated in Figure $8 \mathrm{C}$, produces a clockwise rotation of the edges around their central point. Note that this results in reduction of the edges' distance in the overlapping region. The repulsion acts as illustrated in Figure 8D: here, the edges rotate so that the separation in the overlapping regions increases. This interaction of the edge as a whole is referred to as edge tilt interaction hereafter.

The aim of this experiment was to investigate whether edge interaction is caused by the attraction/repulsion of the component points, illustrated in Figures $8 \mathrm{~A}$ and $8 \mathrm{~B}$, or by a rotation of edge tilt, as illustrated in Figures $8 \mathrm{C}$ and $8 \mathrm{D}$. A new pattern was used. Depicted in Figure 9, it reproduces the induction configuration of McCourt (1982). In Figures 9A and 9B, the background consists of vertical contiguous bands cyclically decreasing/increasing in luminance, while the induced pattern is made up of rows of vertical bars of the same length and luminance. Each row of bars is perceived as rectangular strips made up of close parallel bars, with the horizontal sides consisting of illusory edges formed by collinear bar endings. This pattern usually allows investigation of the brightness induction phenomenon, but we have used it instead to study edge interaction effects. When the bars endings of two adja- cent strips join, the illusory edges do not appear to deviate from linearity (Figure 9A). However, if the distance is changed by displacing the strips (Figure 9B), the contours appear to deform or wave. Since both bar endings and the illusory edge they form are at a close distancethat is, within the interaction range of 3-4 arcmin-we may employ these configurations to explore whether the distorting effects are due to end-to-end local attraction or to edge tilt (both illustrated in Figure 8). Contour distortion changes in accordance with the end-to-end hypothesis are illustrated in Figure 9C, and those in accordance with the edge tilt hypothesis are illustrated in in Figure 9D.

Let us consider the predictions to emerge by assuming that each bar ending is bound to that of its flank to form an illusory edge, so that they follow a common fate in apparent slant. This is whole-edge interaction (edge tilt interaction). The outcomes of the interaction are predictable once we have determined the polarities of contrast in an edge configuration. The outlines in Figure 9B show the contrast polarity of illusory edges formed by the bar endings in two superior strips. Dotted lines illustrate that the interaction occurs between edges of the same contrast polarity $(\mathrm{D} / \mathrm{L})$, since it is these that laterally overlap, and produces a perceived alignment. A clear difference can be seen in the periods of the predicted cyclic waving of the contour according to whether local or whole-edge interaction occurs. The frequency of oscillation on the 


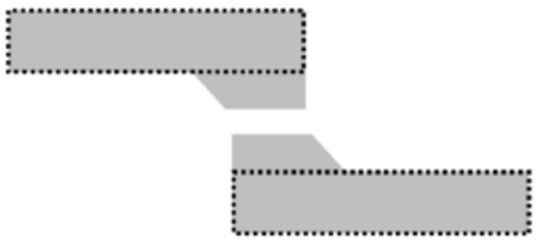

A
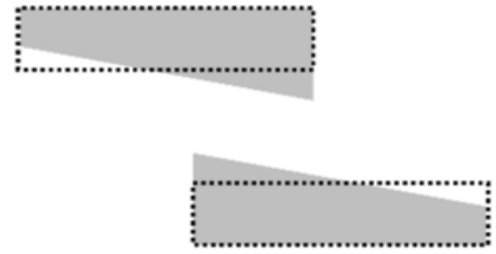

$\mathrm{C}$
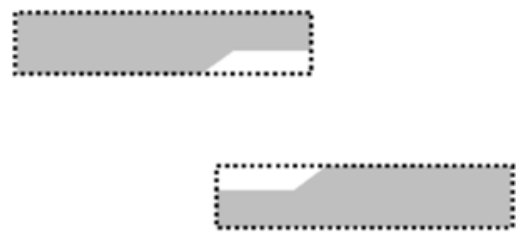

B
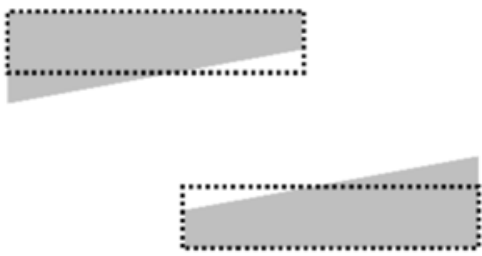

$\mathrm{D}$

Figure 8. Two possible outcomes of edge interaction in relation to direction of contrast. In panels $A$ and $B$, local deformations of two edges of the same (A) or opposite (B) contrast polarity resulting from the attraction (A) and repulsion (B) of points along the flanking edges (end-to-end hypothesis; see the text). In panels $C$ and $D$, the effects of interaction phenomena that affect the whole flanking edges, causing their tilt, are illustrated (edge tilt hypothesis; see the text). A clockwise rotation as in panel $\mathrm{C}$ shortens their distance and is hypothesized to occur with edges of the same contrast polarity; a counterclockwise rotation, as in panel $\mathrm{D}$, has the opposite effect and may be attributed to the interaction of edges of opposite contrast polarity.

basis of the end-to-end hypothesis (Figure 9C) is twice that in Figure 9D. The edge tilt hypothesis predicts synchronous oscillations of background luminance (Figure 9D) and perceived contour. The perceived frequency is, therefore, that predicted by the edge tilt hypothesis.

The set of predictions that can be drawn from the two contrasting hypotheses can be completed if we examine a further case. This is depicted in Figures 10A and 10B, showing vertical segments progressively increasing/ decreasing in luminance and arranged in parallel in horizontal strips against a background of uniform luminance. If we examine, in the outline of Figure 10B, the contrast polarity of the illusory edges formed by the bar endings (D/L or L/D), we see that it is the noncollinear illusory edges of opposite contrast polarity that laterally overlap. This condition is therefore complementary to that depicted in Figure 9B, where interaction is expected to occur between edges of the same contrast polarity. Figure 10B shows edges of opposite contrast polarity that laterally overlap, which are expected to misalign.

In both configurations producing deviation from linearity (Figures 9B and 10B), the perceived oscillation frequency is half that predicted by the end-to-end hypothesis (Figures 9C and 10C) and the same as that predicted by the edge tilt hypothesis (Figures 9D and 10D).

\section{Method}

Subjects. Twenty subjects (12 undergraduate students, 6 postgraduates, and the authors) participated in the experiment. They were randomly assigned to one of two groups.
Stimuli. Two sets of stimuli were prepared.

Set A. The patterns are similar to those depicted in Figures 9A and 9B. Vertical parallel bars of the same length, width, and luminance were drawn at close and regular distances to form a rectangular configuration (strip). Three parallel strips appeared against the background of oscillating luminance: The upper and lower strips had the same luminance; the central strip had the same luminance as the others or a lighter or darker luminance. Six basic configurations were obtained. In four of them, the central strip was lighter or darker, and for each of these conditions two levels of strip luminance, low contrast and high contrast, were used. The luminances of the light and dark bars with high contrast were 78.8 and $8 \mathrm{~cd} / \mathrm{m}^{2}$, and those with low contrast were 52.4 and $18.12 \mathrm{~cd} / \mathrm{m}^{2}$. In the other two basic configurations (controls), the three strips had the same luminance. The strips were $15.1 \mathrm{~mm}$ high and $130 \mathrm{~mm}$ long. Bar thickness was $0.64 \mathrm{~mm}$. The inducing configuration was made up of two periods of a sinusoidal wave. The whole pattern covered a region $130 \mathrm{~mm}$ wide and $83.5 \mathrm{~mm}$ high.

Set $B$. The patterns were similar to those in Set A, with the difference that the luminance changes of the strips and the luminance of the background were homogeneous (Figures 10A and 10B). Bars of equal length and different gray were vertically arranged to form strips of the same dimension as those in Set A. The gray of the bars periodically increased/decreased in luminance, producing two cycles of variation. The stimulus patterns were made up of a central strip and lower and upper strips; the latter were in phase with each other and in counterphase with the central strip. Half of the patterns appeared against a dark gray background $\left(21.8 \mathrm{~cd} / \mathrm{m}^{2}\right)$; the other half appeared against a lighter gray background $\left(72.1 \mathrm{~cd} / \mathrm{m}^{2}\right)$. Four basic patterns were obtained by combining background luminance and cycle of the central strip (from light to dark or from dark to light), and the other two, the control patterns, had three strips of the same phase.

The stimuli were computed by the MCDraw program and were presented on an Apple Multiple Scan 15 display. 
Procedure. The procedure was the same for the two groups, one performing the experiment with Set A stimuli, the other with Set B stimuli. In each of the stimuli and for each of the 6 configurations, 13 configurations were generated by varying, in $0.32-\mathrm{mm}$ steps, the distance between the strips from 0 to $3.8 \mathrm{~mm}$. The 13 patterns were presented to the subjects once in increasing order and once in decreasing order of vertical distance between the strips. The observer was presented with a first pattern having the strips at largest separation or aligned with the extremes. A pattern then followed in which the distance between the contours was shortened or lengthened.

The subjects were instructed to observe the region between the central and the contiguous strips and report whether they perceived an oscillating instead of a linear strip. If so, they were asked to inspect the central strip and to indicate the positive peaks of the wave. Some practice trials followed.

For each subject, the experiment was repeated twice with 120and $180-\mathrm{cm}$ viewing distance, in random order. Each subject completed one set of trials at one distance and then initiated the set at the higher (lower) distances. The initial viewing distance was chosen randomly for each subject.

\section{Results}

We have two types of data: the perceived phase of the waving and the extension of the region within which it was observed. These results will be examined separately.

Set A. Perceived phase is easy to report, since all the subjects described a contour oscillation (when perceived) in line with period and phase predictions depicted in Figure 9D-that is, predictions generated by the edge tilt hypothesis. Table 6 reports the $D_{\min }$ and the $D_{\max }$ values at which illusory waving was observed. None of the control patterns produced misperception. Separate analyses of variance for the $D_{\min }$ and the $D_{\max }$ values were carried out, yielding the following variables: viewing distance, contrast between strips, and central strip luminance (darker vs. lighter). The viewing distance affected the $D_{\max }$ values $[F(1,9)=11.83, p<.01]$. Significantly different $D_{\min }$ values were registered in the two contrast levels: [low contrast, 1.21 arcmin; high contrast, 1.50 arcmin; $F(1,9)=14.95, p<.005]$.

Set $B$. The observed oscillations are in agreement with the hypothesis that edges of opposite contrast polarity tilt so as to appear at a greater distance than the actual value. The periodical waving indicated by the subjects is in line with the period and phase predictions of the edge tilt hypothesis schematized in Figure 10D, and not with those predicted by the end-to-end hypothesis (Figure 10C). Separate analyses of variance were carried out for $D_{\min }$ and $D_{\max }$ values. Both analyses considered factors of viewing distance, ground luminance (dark vs. light), and central strip phase (central peak darker vs. lighter). The $D_{\max }$ values were found to be affected by viewing distance $[F(1,9)=6.83, p<.05]$, as well as the $D_{\min }$ values $[F(1,9)=14.22, p<.01]$; the values are summarized in Table 7 . The $D_{\min }$ values yielded higher values $(.98$ vs. .80 arcmin $)$ with a darker background $[F(1,9)=$ $27.02, p<.001]$.

\section{Discussion}

The ranges of spatial interaction shown are similar to those registered in Experiments 1 and 2. The dependence of direction of misplacement on the contrast polarity agrees with the results of Experiment 2. Experiment 3 confirms that the "units" of spatial interactions are, in this particular context, the edges, thus leading to the conclusion that edge formation precedes, or is contemporary with, the interaction phenomena that arise between simpler units, such as points or line endings. This finding is not accounted for by a popular model of integration (Grossberg \& Mingolla, 1985) of collinear contours, suggesting that different mechanisms for collinear and noncollinear edges exist.

\section{GENERAL DISCUSSION}

Experiment 1 shows that when an edge can be perceived to continue either with a collinear edge of the opposite contrast polarity or with a noncollinear edge of the same contrast polarity, observers perceive an alignment between edges of the same polarity, even though they are noncollinear. Experiment 2 shows that two noncollinear edges appear to be aligned when they have the same contrast polarity but misaligned when they have opposite contrast polarity. In Experiment 3, we set in opposition two alternative explanations of these effects. The first follows from the well-known finding of a shift of perceived position (attraction and repulsion) between closely presented target contours (Badcock \& Westheimer, 1985b; Hotopf \& Brown, 1988; Rentschler et al., 1975), which predicts local interactions, either end to end or point to point, where they laterally overlap. The second explanation also assumes a spatial interaction between two noncollinear contours but, differently from the attraction/repulsion hypothesis, predicts that the interaction regards the edges, or part of them, and produces an inward tilt (so that the edges with the same contrast polarity perceptually align) or an outward tilt (so that edges of opposite contrast polarity are perceived to be more misaligned). The results of Experiment 3 rule out the local attraction/repulsion explanation and, instead, support the edge tilt explanation.

Moreover, the effect of our independent variables (contrast, viewing distance, and luminance profile of the configurations used) on the dependent variables (distance between two noncollinear edges at which the illusion occur) gives some insight into the underlying mechanism. First, all the experiments show that, regardless of configuration, there is a tendency to perceive two noncollinear laterally overlapping as aligned, providing that the displacement between the two edges is larger than about $1 \operatorname{arcmin}\left(D_{\min }\right)$ or smaller than 3-4 $\operatorname{arcmin}\left(D_{\max }\right)$.

This $D_{\min }$ value is similar to thresholds for positional change (De Valois \& De Valois, 1988; Westheimer \& McKee, 1977), whereas the $D_{\max }$ limit is similar to that found between test and inducing elements (lines or edges) in both perceived position judgment and contrast sensitivity. For briefly presented stimuli, attraction effects have been observed between inducing and test lines both darker than the background and separated by no 

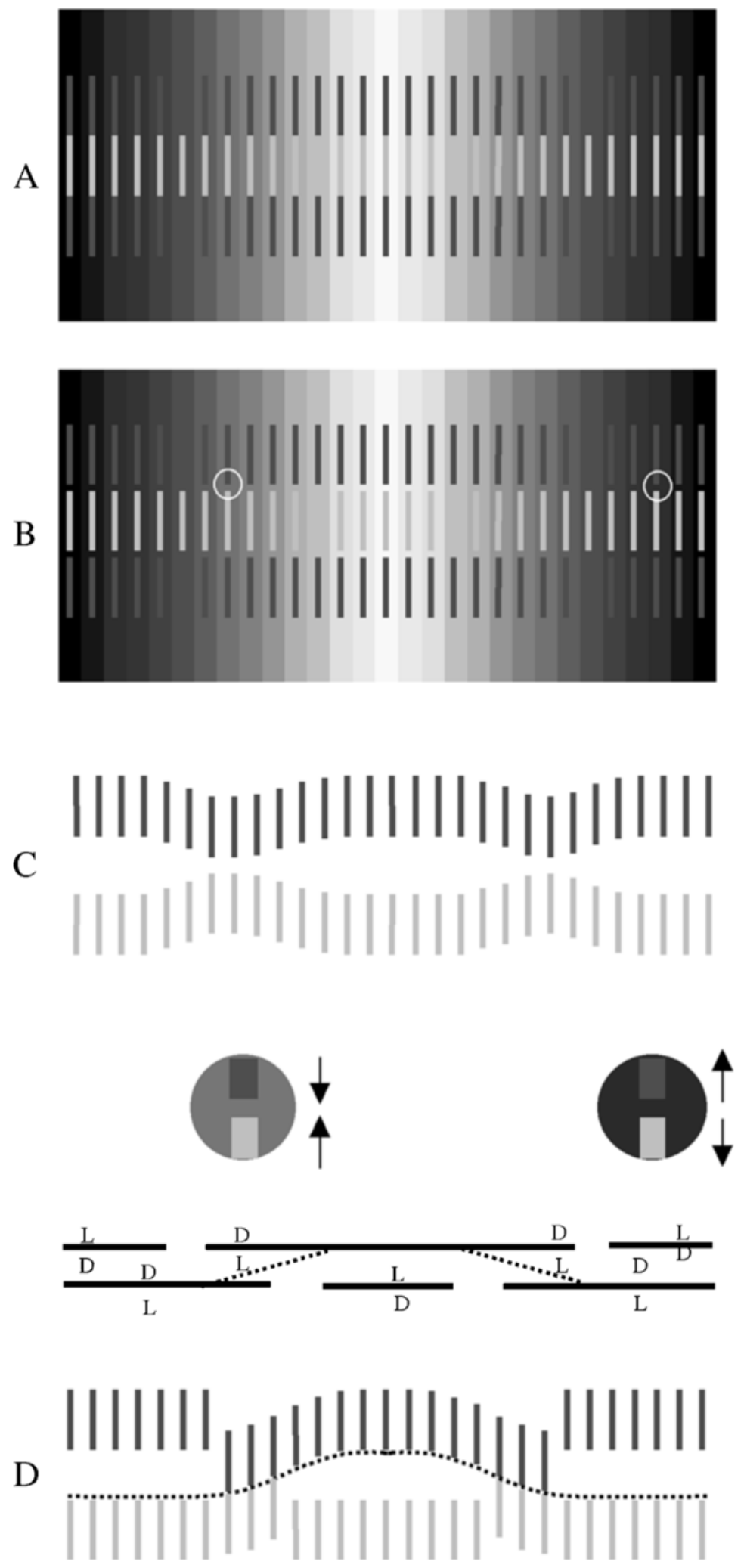

Figure 9. Figure caption appears on the following page. 
Figure 9. Vertical bars of the same length and luminance, arranged in three horizontal strips placed on a background consisting of a sinusoidal luminance grating. The strips are vertically separated by a short distance in panel $B$ and are not separated in panel A. Circular shapes show, enlarged, the circled areas in panel $B$ and the bar endings inside. Arrows indicate the attraction/repulsion interactions resulting from contrast polarity. The outlines symbolize the illusory edges formed by the bar endings of the upper and the middle strips. Panel $C$ schematizes, magnifying the effects, the distortions predicted by the hypothesis that the interactions between the bar endings in panel $B$ consist in point-to-point attraction/repulsion. Panel D schematizes the hypothesis that the interaction affects the whole illusory edges formed by aligned line terminators.

more than 3-4 arcmin (Badcock \& Westheimer, 1985b), but also repulsion effects have been observed within larger spatial limits. Similar effects were registered by Hock and Eastman (1995) with inducing and test lines in close succession. Within this short range, Badcock and Westheimer (1985a) registered repulsion effects between a test line and an induction pattern made up of two collinear segments separated for a length equal to the test line extension. ${ }^{1}$ With separations greater than 3-4 arcmin, only repulsion was observed, independently of the test and inducing lines contrast polarity. In a localization task, Greene and Brown (1997) registered both weak attraction and weak repulsion effects with illusory lines separated 4-9 arcmin; when the distance was shortened below 3 arcmin, they observed a light flank attraction of both light and dark illusory contour targets and a dark flank repulsion (or weak attraction), irrespective of target lightness. Also, no clear indication emerged as regards the relation between contrast polarity and apparent displacement in experiments testing the interaction between adjacent boundaries (edges). The flank attraction was observed in both directions of lightness (Greene \& Brown, 1995; Rivest \& Cavanagh, 1996). One of the results obtained by Greene and Brown (1995) is of particular relevance to the present results. For high-contrast flanks with a separation of 3.69 arcmin, negative polarity of flanking boundary shows stronger and wider attraction effects than positive polarity, a result consistent with that observed in our experiments with edges of the same contrast polarity. Other research revealed interaction ranges beyond this limit (Hotopf \& Brown, 1988; Rentschler et al., 1975; Rivest \& Cavanagh, 1996; see also Polat \& Sagi, 1994, in research on contrast sensitivity), but the hypothesis that locates the stronger interaction phenomena within this short-range limit appears to be sufficiently well founded.

Our data are thus in agreement with most work with regard to width of region interaction: When two patterns (lines or boundary) are located at a distance shorter than 3-4 arcmin in visual angle, they appear to be displaced. Nevertheless, our data disagree with those previously reported in two important aspects: First, we do not replicate findings showing a relationship between distance and effect of contrast polarity; second, there are no pre- vious reports of a relation between contrast polarity and direction of apparent shift, which emerged in our experiments. For these reasons, the models generated to account for attractive/repulsive spatial interactions are not suitable to interpret our results. Instead, the candidate model must explain how the perceptual misalignment occurs and why the apparent misplacement takes different directions in relation to the contrast polarity of the nearby edges. To answer these questions, several considerations have to be addressed.

1. Although contrast seemed to affect $D_{\max }$ and $D_{\text {min }}$ (mainly in Experiment 1$)$, the spatial separation $\left(D_{\max }-\right.$ $\left.D_{\min }\right)$ producing perceived edge tilt was almost fixed in all the experiments $(M=2.29 \operatorname{arcmin}, S D=0.35)$. This spatial separation of constant amplitude occurs despite the difference in stimuli configuration and type of interacting contour used.

The finding that there is almost a fixed range of spatial interactions suggests that the underlying mechanism presents position specificity. Both position selectivity and selectivity for contrast polarity are properties of a very early stage of processing of the central visual system. De Valois and De Valois (1988) reported that, in the striate cortex, phase-selective simple cells tuned to high spatial frequency modulate their responses in relationship to very small position shifts. Instead, complex cells insensitive to contrast polarity are less sensitive to position shift.

2 . The second consideration concerns the spatial constraints for the illusion to occur, summarized in Figure 11. The diagrams symbolize the typical spatial arrangement of noncollinear laterally overlapping edges, with the same (Figure 11A) and opposite (Figure 11B) contrast polarity, their corresponding luminance profile, and the direction of perceived distortion in the two cases (see segments T1 and T2). Experiments $1 \mathrm{~A}$ and 2 show that lateral overlapping is an important constraint for the edges to join perceptually. Figure 11A illustrates, with edges of the same contrast polarity, that this may happen

Table 6

Mean Values (in Arcmin) of $D_{\min }$ and $D_{\max }$ Distances in Relation to Viewing Distance for the Waving Effect to be Perceived in Set A, Experiment 3

\begin{tabular}{cccc}
\hline $\begin{array}{c}\text { Viewing Distance } \\
(\mathrm{cm})\end{array}$ & $D_{\min }$ & $D_{\max }$ & $D_{\max }-D_{\min }$ \\
\hline 120 & 1.47 & 4.28 & 2.81 \\
180 & 1.24 & 3.54 & 2.30 \\
\hline
\end{tabular}

Table 7

Mean Values (in Arcmin) of $D_{\min }$ and $D_{\max }$ in Relation to Viewing Distance for the Waving Effect to be Perceived in Set B, Experiment 3

\begin{tabular}{cccc}
\hline $\begin{array}{c}\text { Viewing Distance } \\
(\mathrm{cm})\end{array}$ & $D_{\min }$ & $D_{\max }$ & $D_{\max }-D_{\min }$ \\
\hline 120 & 1.02 & 3.07 & 2.05 \\
180 & 0.76 & 2.72 & 1.96 \\
\hline
\end{tabular}


A

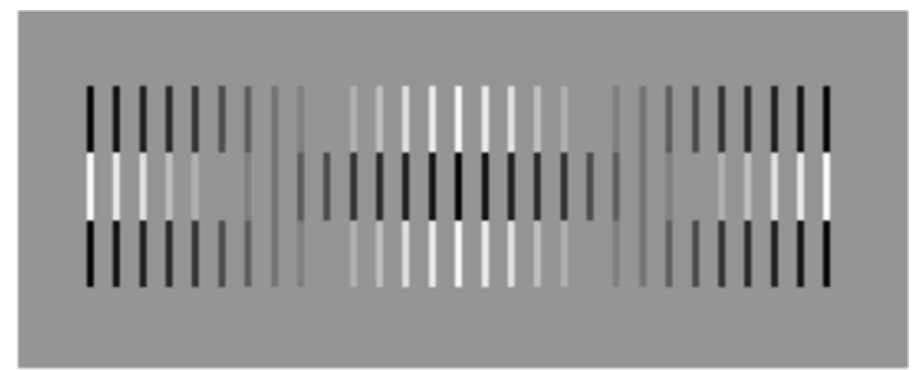

B

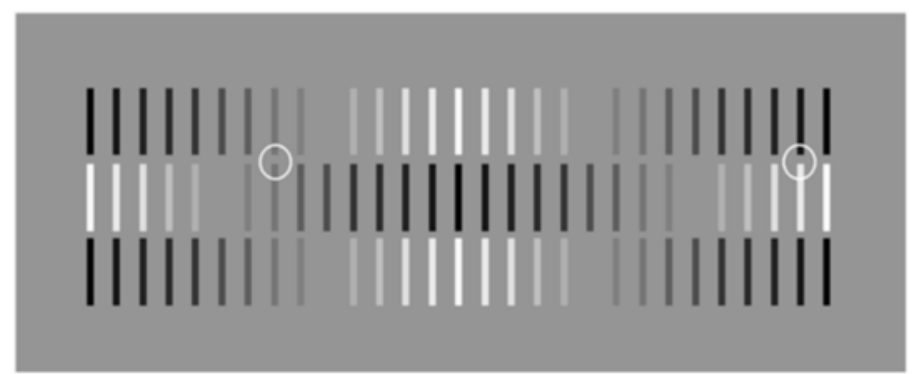

C
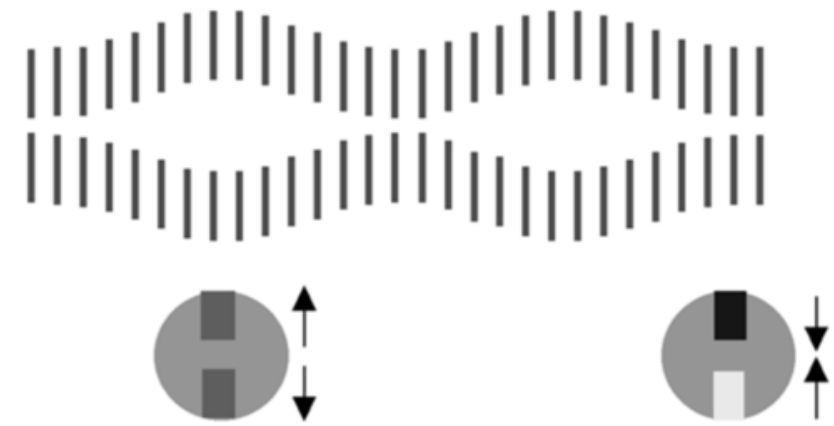

D
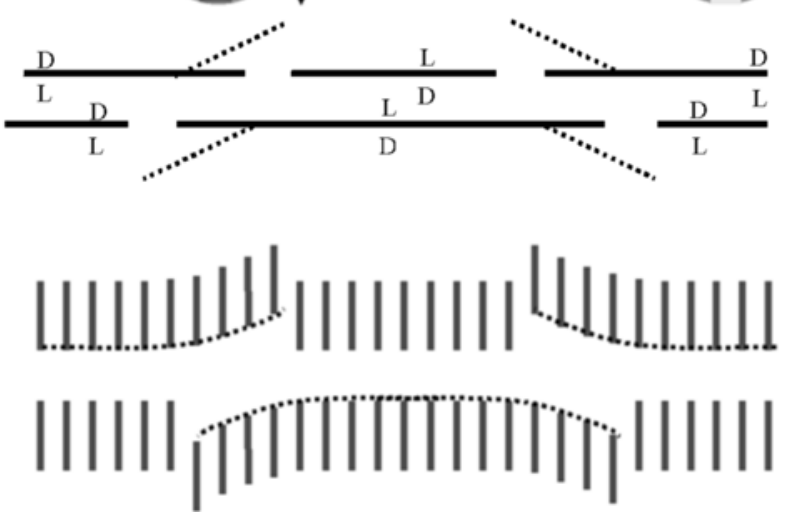

Figure 10. Bars of periodically changing luminance placed on a uniform luminance background. The strips are vertically separated in panel $B$ and are not separated in panel A. Circular shapes show, enlarged, the circled areas in panel $B$ and the bar endings inside. Arrows indicate the attraction/repulsion interactions resulting from contrast polarity. The outlines symbolize the illusory edges formed by the bar endings of the upper and the middle strips. Panel $\mathrm{C}$ schematizes, magnifying the effects, the distortions predicted by the hypothesis that the interactions between the bar endings in panel $B$ consist in point-topoint attraction/repulsion. Panel D schematizes the hypothesis that the interaction affects the whole illusory edges formed by aligned line terminators. 
because, between nonoverlapping edges $\mathrm{L} / \mathrm{D}$, there is a gap with a portion of edges with opposite contrast polarity (D/L). Clearly, in this case, the contrast polarity rule accounts not only for the perceived alignment between two noncollinear edges, but also for which of two pairs of noncollinear edges will tend to align-that is, the directions of binding.

3. Increasing the difference in luminance (contrast) increases the region of overlap between the two D/L edges. In Table 2, we observe that high-contrast values corresponding to larger $\mathrm{O}$ values are paired with larger $D_{\max }-$ $D_{\text {min }}$ magnitudes. However, our results show that these two parameters are not directly related. On the contrary, as contrast increases, $D_{\max }-D_{\min }$ is almost fixed. Moreover, Experiment 1A shows that, if the amount of overlap is varied independently for two levels of contrast, overlap threshold does not depend on contrast. These findings clearly indicate that relative luminance is a cue for triggering the binding mechanism and determining the direction of binding (clockwise vs. counterclockwise) but does not affect the distance at which binding occurs, as the results of Experiment 1A show. Following this argument, it appears that only the end segments of the edges participate in the binding process for a particular difference of luminance. This interpretation is consistent with the common suggestion in the literature that linear stimulus can be defined functionally, either psychophysically (Yu \& Essock, 1996) or neurophysiologically (Orban, Kato, \& Bishop, 1979), by the end segments of a linear stimulus. Our hypothesis is that there is a fixed portion of two noncollinear edges, coincident with their end segments (indicated as T1 and T2 in Figures $11 \mathrm{~A}$ and $11 \mathrm{~B}$ ), that are perceived as tilted so that they perceptually align (as illustrated in Figure 11A) or misalign with respect to the physical misalignment (Figure 11B), depending on the contrast polarity of the two edges. The results of Experiment $1 \mathrm{~A}$ indicate that the length of these segments is, at an average separation between the noncollinear edges, equal to $7.27 \mathrm{arcmin}$. The tilt interpretation is in agreement with our assumption that the underlying mechanism responsible for tilting end segments operates at a very early stage of processing in the central visual system. Indeed, as we have discussed, our results support the assumption of position and contrast polarity selectivity, which are properties of these early stage mechanisms. Since these early mechanisms are also selective for orientation, they may well underlie the tilt effect. Indeed, simple cells present a median half-height bandwidth of $\pm 21^{\circ}$ (De Valois, Yund, \& Hepler, 1982), so that a cell activated by an edge horizontally oriented, such as those in Figure 11, would also respond to an orientation close to horizontal. Our conjecture is that the tilt assumed by aligning edges is compatible with the orientation bandwidth of early mechanisms. Since alignment occurs within a fixed separation of $2.36 \mathrm{arcmin}$, and since the mean extent of edge portion which undergoes a distortion $(\mathrm{K})$ is about 7.27 arcmin
(Experiment 1A), $\alpha$ can be easily calculated with the equation $\alpha=\arctan (\operatorname{separation} / \mathrm{K})$; its magnitude is $17.9^{\circ}$. This value of $\alpha$ is very compatible with the orientation bandwidth of early contrast-sensitive cortical mechanisms.

4. Not only do two noncollinearedges having the same contrast polarity appear as aligned, but also two noncollinear edges with opposite polarity appear to be more misaligned than they in fact are. How can the effect of misalignment be reconciled with our suggestion that the effect is based on the activation of an early mechanism? We suggest that early mechanisms sensitive to contrast polarity could account for the finding that the perceived tilt is inward in the top configuration of Figure 11 (same contrast polarity of the two noncollinear edges is different) and outward in the configuration at the bottom (different contrast polarity). To account for this opposite direction of tilt, it is sufficient to assume that, since the luminance profiles of the two luminance configurations are different (see the two outline profiles on the right of Figures 11C and 11D), filters with different receptive fields are activated. Indeed, a filter with odd-receptive field will be better stimulated by a pattern with the luminance profile in Figure 11C. On the other hand, an even symmetric receptive field will be better stimulated by a pattern with the luminance in Figure 11D. It may be hypothesized, following Badcock and Westheimer (1985b, p. 1263), that the gray strip in Figure 11D may be treated by the visual system as a single entity in the region in which it is embedded within two lighter region, a condition that inhibits the emergence of the T-endings. The hypothesized effects are symbolized in Figures 11C and $11 \mathrm{D}$ by solid lines for activated T-endings and dotted lines for inhibited T-endings.

5. An explanation is also needed of why these filters respond for an orientation different from the physical one. This result can also be compatible with the activation of early mechanisms. The hypothesis that early mechanisms may respond to illusory contours, as well as to physical ones, has received strong support from the physiological data of Peterhans and von der Heydt (1993).

It is worth considering our results in the light of the more popular models of contour integration. Our results are not in line with those predicted by models of binding of collinear contours (Grossberg \& Mingolla, 1985), suggesting that our mechanism is specific for the binding of noncollinear contours. Our results, however, are not incompatible with the idea that contour integration is achieved on the basis of an association of response of oriented filters activated by ends of the spatially interacting edges (Field et al., 1993, 2000), which is also in agreement with the observations that these authors have made that the ability to integrate contours along a path depends on contrast polarity.

It would also be interesting to relate our effects of perceived shape of noncollinear spatial interaction contours 
1270 RONCATO AND CASCO
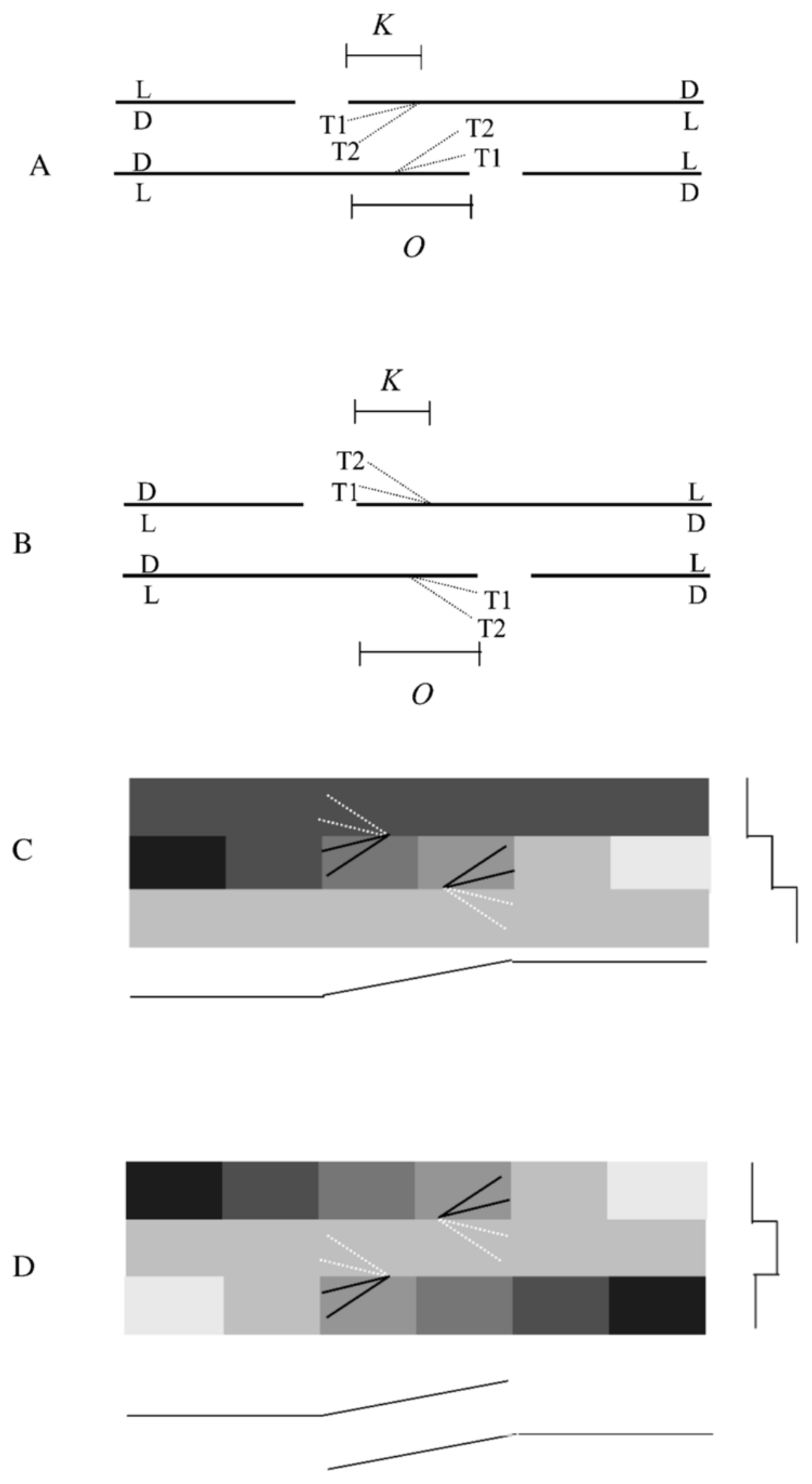
Figure 11. The two outline patterns illustrate a condition of edges of the same contrast polarity (A) and different contrast polarity $(B)$ running parallel alongside. $O$ indicates the overlapping region, and $K$ indicates the region in which the extreme parts of the edges may be misperceived in orientation. The direction of luminance change is indicated by dark/light $(\mathrm{D} / \mathrm{L})$ or light/dark (L/D) at the extremes. T1 and T2 indicate the end portion of a line that underlies a tilt. The patterns in panels $C$ and $D$ are examples of the luminance combinations schematized in panels $A$ and $B$, respectively, and on the right of these patterns, the outline illustrates the luminance profiles following a vertical section of the $K$ region. The segments irradiating from a point near the edge endings symbolize the different orientations of tilt in which the extreme may be perceived. The dotted lines indicate orientations that, according to the hypothesis illustrated in the text, are most unlikely to be perceived. The outlines below the patterns in panels $C$ and $D$ illustrate the perceptual outcome due to the edge orientation misperception.

to those involved in the perception of the brightness of surfaces. Apparently, these are different phenomena, since brightness perception seems to depend on contrast in a different way than perceived contour slant does. For example, it has been shown (Reid \& Shapley, 1988) that local contrast is very important in brightness perception, since it determines brightness induction-that is, it makes an object appear brighter on a background of lower luminance and darker on a background of greater luminance. Similarly, Dresp and Fischer (2001) showed that contrast, rather than contrast polarity, is the important factor in the integration of inducing stimuli in a Kaniza's square configuration to produce perceptual filling in. On the contrary, we have shown the contrast has only a limited role in the spatial interaction between noncollinear edges. Therefore, it seems that the perceived shape of noncollinear contours and brightness perception occur at a different level of visual processing: Contour integration occurs at a low level of processing where polarity of contrast is used as information, whereas perceptual filling (and maybe brightness perception) occurs at a later stage of integration of both luminance contrast and spatial information that discards information on contrast polarity or color.

To conclude, it is worth considering that the present article goes beyond an attempt to define the local mechanism responsible for edge distortion. Through the use of complex patterns such as those in Experiments 2 and 3 , we have aimed to give a phenomenological demonstration that when these local distortions are repeated many times in a configuration, they can produce overall effects that can be understood as a sum of local effects. We are now running psychophysical objective tests of these phenomenological observations. Preliminary results show that it is generally true that local deformations, such as those modeled in Figure 11, may sum to account for global contour deformation effects. It seems likely that global deformation is a consequence of the activation of phase-dependent collector units oriented with the illusory local orientation, which may "link" pairs of horizontally contiguous edges and account for overall distortions.

\section{REFERENCES}

Badcock, D. R., \& Westheimer, G. (1985a). Spatial location and hyperacuity: Flank position within the centre and surround zones. Spatial Vision, 1, 3-11.

BADCock, D. R., \& WeStheimer, G. (1985b). Spatial location and hyperacuity: The centre/surround localization contribution function has two substrates. Vision Research, 25, 1259-1267.

Bex, P. J., \& EDGar, G. K. (1996). Shifts in the perceived location of a blurred edge increase with contrast. Perception \& Psychophysics, 58, 31-33.

Cohen, M. A., \& Grossberg, S. (1984). Neural dynamics of brightness perception: Features, boundaries, diffusion, and resonance. Perception \& Psychophysics, 36, 428-456.

De Valois, R. L., \& De Valois, K. K. (1988). Spatial vision. New York: Oxford University Press.

De Valois, R. L., Yund, E. W., \& Hepler, N. (1982). The orientation and direction selectivity of cells in macaque visual cortex. Vision Research, 22, 531-544.

Dresp, B. (1999). Dynamic characteristics of spatial mechanisms coding contour structures. Spatial Vision, 12, 129-142.

DresP, B., \& Fischer, S. (2001). Asymmetrical contrast effects induced by luminance and color configurations. Perception \& Psychophysics, 63, 1262-1270.

DresP, B., \& Grossberg, S. (1997). Contour integration across polarities and spatial gaps: From local contrast filtering to global grouping. Vision Research, 37, 913-924.

Field, D. J., Hayes, A., \& Hess, R. F. (1993). Contour integration by the human visual system: Evidence for a local "association field." $\mathrm{Vi}$ sion Research, 33, 173-193.

Field, D. J., Hayes, A., \& Hess, R. F. (2000). The roles of polarity and symmetry in the perceptual grouping of contour fragments. Spatial Vision, 13, 51-66.

Finney, D. J. (1971). Probit analysis. Cambridge: Cambridge University Press.

Gilchrist, I. D., Humphreys, G. W., Riddoch, M. J., \& Neumann, H. (1997). Luminance and edge information in grouping: A study using visual search. Journal of Experimental Psychology: Human Perception \& Performance, 23, 464-480.

Greene,H. H., \& Brown, J. M. (1995). The effect of nearby luminance contrast polarity on colour boundary localization. Vision Research, 35, 2767-2771.

Greene, H. H., \& Brown, J. M. (1997). Spatial interactions with real and gap-induced illusory lines in vernier acuity. Vision Research, 37, 597-604.

Grossberg, S., \& Mingolla, E. (1985). Neural dynamics of perceptual grouping: Textures, boundaries, and emergent segmentations. Perception \& Psychophysics, 38, 141-171.

HE, Z J., \& OoI, T. L. (1998). Illusory-contour formation affected by luminance contrast polarity. Perception, 27, 313-335.

Hock, H. S., \& EASTMAN, K. E. (1995). Context effects on perceived position: Sustained and transient temporal influences on spatial interactions. Vision Research, 35, 635-646.

Ноторғ, W. H., \& Brown, S. A. (1988). Constant errors in judgements of collinearity due to the presence of neighbouring objects. Perception, 17, 523-534.

Kellman, P. J., \& Shipley, T. F. (1991). A theory of visual interpolation in object perception. Cognitive Psychology, 23, 141-221.

McCourt, E. M. (1982). A spatial frequency dependent grating-induction effect. Vision Research, 22, 119-134.

McIlHagGa, W. H., \& Mullen, K. T. (1996). Contour integration with colour and luminance contrast. Vision Research, 36, 1265-1279.

Orban, G. A., Kato, H., \& Bishop, P. O. (1979). End-zone region in receptive fields of hypercomplex and other striate neurons in the cat. Journal of Neurophysiology, 42, 818-832.

Peterhans, E., \& von der Heydt, R. (1993). Functional organization 
of area V2 in the alert macaque. European Journal of Neuroscience, 5, 509-524.

PolAT, U., \& SAGI, D. (1994). The architecture of perceptual spatial interactions. Vision Research, 34, 73-78.

REID, R. C., \& Shapley, R. (1988). Brightness induction by local contrast and the spatial dependence of assimilation. Vision Research, 28, 115-132.

Rentschler, I., Hilz, R., \& Grimm, W. (1975). Processing of positional information in the human visual system. Nature, 253, 444-445.

Rivest, J., \& Cavanagh, P. (1996). Localizing contours defined by more than one attribute. Vision Research, 36, 53-66.

Wehrhahn, C., \& Dresp, B. (1998). Detection facilitation by collinear stimuli in humans: Dependence on strength and sign of contrast, $\mathrm{Vi}$ sion Research, 38, 423-428.
Westheimer, G., \& McKee, S. P. (1977). Integration regions for visual hypercuity. Vision Research, 17, 89-93.

Yu, C., \& Essock, E. A. (1996). Psychophysical end-stopping associated with the line target. Vision Research, 36, 2883-2896.

\section{NOTE}

1. Since we measured the contrast polarity by following a perpendicular to the two profiles, and not from the figures to the common ground, as in Badcock and Westheimer (1985a), these effects of contrast polarity are opposite to the results we registered in the present experiments.

(Manuscript received January 29, 2002; revision accepted for publication April 21, 2003.) 